\title{
Influence of loudspeaker directivity and measurement geometry on direct acoustic levels over façades for acoustic insulation tests with the International Standard ISO 140-5
}

\author{
José Luis Sánchez Bote , Antonio Pedrero González, Juan José Gómez Alfageme
}

\begin{abstract}
A B S T R A C T
The International Standard ISO $140-5$ on field measurements of airborne sound insulation of façades establishes that the directivity of the measurement loudspeaker should be such that the variation in the local direct sound pressure level $(\triangle \mathrm{SPL}$ ) on the sample is $\Delta \mathrm{SPL}<5 \mathrm{~dB}$ (or $\Delta \mathrm{SPL}<10 \mathrm{~dB}$ for large façades). This condition is usually not very easy to accomplish nor is it easy to verify whether the loudspeaker produces such a uniform level. Direct sound pressure levels on the ISO standard façade essentially depend on the distance and directivity of the loudspeaker used. This paper presents a comprehensive analysis of the test geometry for measuring sound insulation and explains how the loudspeaker directivity, combined with distance, affects the acoustic level distribution on the façade.

The first sections of the paper are focused on analysing the measurement geometry and its influence on the direct acoustic level variations on the façade. The most favourable and least favourable positions to minimise these direct acoustic level differences are found, and the angles covered by the façade in the reference system of the loudspeaker are also determined. Then, the maximum dimensions of the façade that meet the conditions of the ISO 140-5 standard are obtained for the ideal omnidirectional sound source and the piston radiating in an infinite baffle, which is chosen as the typical radiation pattern for loudspeakers.

Finally, a complete study of the behaviour of different loudspeaker radiation models (such as those usually utilised in the ISO 140-5 measurements) is performed, comparing their radiation maps on the façade for searching their maximum dimensions and the most appropriate radiation configurations.
\end{abstract}

\section{Introduction}

This paper discusses and analyses the requirements of the International Standard ISO 140-5 [1-3] with respect to the characteristics of the emitter loudspeaker and its relation to the measurement geometry, as described in this standard. The standard ISO 140-5: 1998 in Part 5, that is: "Field measurements of airborne sound insulation of façade elements and façades", describes various methods for measuring airborne noise insulation of whole façades (global methods) and façade elements, such as a window (element methods); the methods that are particularly interesting in this paper are those using a loudspeaker as a noise source, as follows:

- The element loudspeaker method: the acoustic level on the outer façade is measured by averaging different positions of a microphone placed flush with the façade. The distance from the loudspeaker to the surface centre must be at least $5 \mathrm{~m}$.
- The global loudspeaker method: the outside acoustic level is measured at a single point with a microphone placed at $2 \mathrm{~m}$ from the centre of the façade (distance loudspeaker-façade centre at least $7 \mathrm{~m}$ ).

This text uses the term "façade" to refer both to the whole façade and the façade element because this difference is not substantial for the measurement geometry analysed here and the conclusions can be applied to both the global and the element method, whenever loudspeakers are the sound source.

Section 4.2 of the ISO standard outlines the requirements to be met in terms of loudspeaker directivity, which will receive special attention in this paper. Specifically, the loudspeaker should ensure that the local differences of sound pressure levels ( $\triangle$ SPLs) on the façade in all frequency bands of interest are less than $\Delta S P L=5 \mathrm{~dB}$, measured in free field on a surface that is the same size and orientation as the wall or element to be tested. If the loudspeaker method is implemented with large area samples (e.g., exceeding $5 \mathrm{~m}$ ), differences of up $\Delta S P L=10 \mathrm{~dB}$ can be accepted. This requirement specifies the uniformity level conditions that must be ensured on 
the façade under study, not necessarily in the test moment, but under ideal free acoustic field conditions, either in a separate measurement or in a simulation (as is proposed in the paper), because the free field restriction will not normally be met by the façade in its typical arrangement.

Additionally, point 5.3 of the standard refers to the "generation of sound field", proposing that at least the third octave bands with centre frequency bands from $100 \mathrm{~Hz}$ to $3150 \mathrm{~Hz}$ be used (preferably from $50 \mathrm{~Hz}$ to $5000 \mathrm{~Hz}$ ). Eventually, the loudspeaker position and aim are stated, which are characteristics to be discussed in detail below.

In practice, the uniformity level condition on the façade depends on the loudspeaker directivity and position and the dimensions of the façade, but this uniformity condition is difficult to estimate a priori. Some laboratories measure the sound field generated on an imaginary surface of the same dimensions as the façade being tested, either situated in an anechoic room or in an outside location, assuming high acoustic absorption of the ground, which is always present. Neither of these measurement procedures is ideal. The anechoic room is usually too small for realistic façade sizes, and the outside ground is not as absorbent as required. If the measurement geometry of the test according to ISO $140-5$ and the loudspeaker directivity are known, then the distribution of direct acoustic levels on any surface as set by the standard can be realised. This paper presents a comprehensive analysis of the test geometry in agreement with the ISO 140-5 standard, and this analysis is combined with the radiation patterns of several models of loudspeakers typically used in such sound insulation measurements.

Chapter two discusses the test geometry, and in chapter three, the previous results are related to the theoretical directivity function of a piston. In chapter four, direct acoustic level simulations are performed on various façade specimens and with different sound source models, and finally, chapter five is devoted to the conclusions.

\section{Influence of measurement geometry and loudspeaker position on acoustic level differences}

According to ISO $140-5$, the loudspeaker should be placed far enough from the façade to minimise the variation of sound pressure levels on the test sample. Although the sound source is preferably placed on the floor, alternatively, it can be located as high from the ground as possible. Fig. 1 shows a geometric model con-

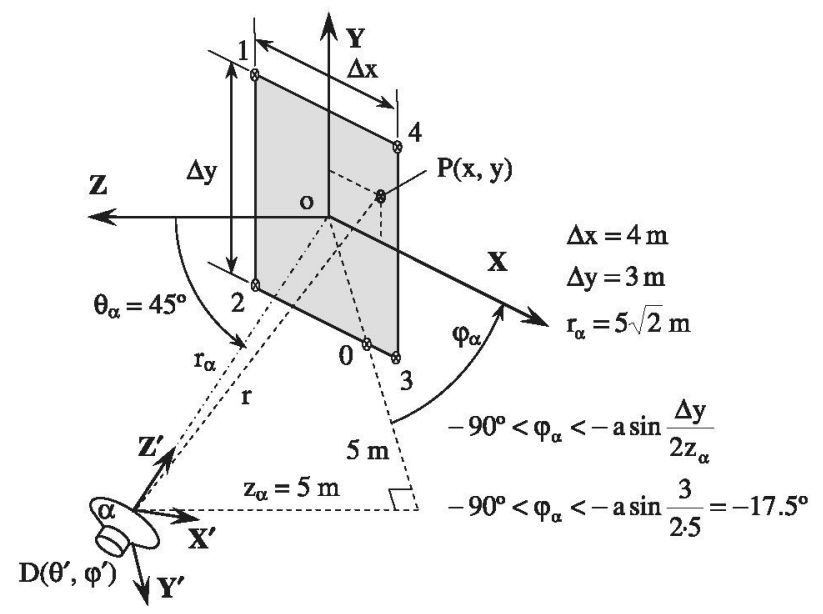

Fig. 1. Test geometry and loudspeaker position. Particularisation to a standard façade with $\Delta x \times \Delta y=4 \mathrm{~m} \times 3 \mathrm{~m}$ and the sound source at $r_{\alpha}=5 \sqrt{ } 2=7 \mathrm{~m}\left(z_{\alpha}=5 \mathrm{~m}\right)$ from the coordinate and surface centre.

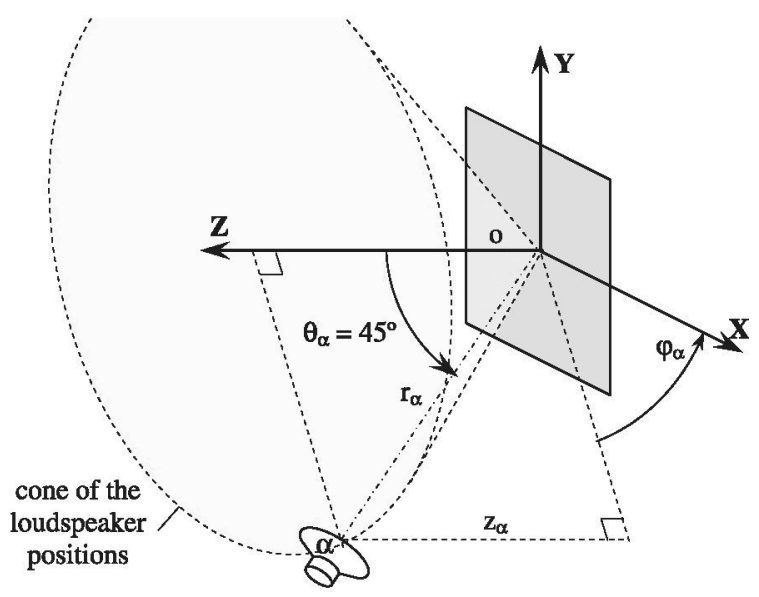

Fig. 2. Cone of the loudspeaker positions.

sistent with the ISO standard. According to this model, the origin "o" is placed at the centre of the façade. Therefore, the unitary vectors $\mathbf{X}, \mathbf{Y}$ and $\mathbf{Z}$; or $\mathbf{r}, \boldsymbol{\theta}$ and $\boldsymbol{\varphi}$, respectively, define the axis system associated with both Cartesian and spherical coordinates. The $\mathbf{Z}$ axis is chosen perpendicular to the façade, as this position simplifies the study in spherical coordinates because the elevation angle $\theta_{\alpha}$ of the loudspeaker remains constant due to the ISO standard requirements. The façade has a rectangular shape with width $\Delta x$ and height $\Delta y$. The loudspeaker " $\alpha$ " is placed before the façade at the point $\left(r_{\alpha}, \theta_{\alpha}, \varphi_{\alpha}\right)^{1}$ expressed in spherical coordinates, and pointing towards the centre of the façade (although this detail is not mandatory). The function $D\left(\theta^{\prime}, \varphi^{\prime}\right)$ is the loudspeaker directivity, which represents the normalised sound pressure gain (conventionally with respect to the axis of the source) depending on the azimuth and elevation angles $\left(\theta^{\prime}, \varphi^{\prime}\right)$, measured in the reference system of the measurement loudspeaker.

Following the ISO standard, the sound incidence angle should be $\theta_{\alpha}=45 \pm 5^{\circ}$. Therefore, it holds that $r_{\alpha}=\sqrt{ } 2 z_{\alpha}$. The distance from the sound source to the sample centre should also meet the conditions $r_{\alpha}>5 \mathrm{~m}\left(z_{\alpha}>3.5 \mathrm{~m}\right)$ in the case of the element loudspeaker measurement method and $r_{\alpha}>7 \mathrm{~m}\left(z_{\alpha}>5 \mathrm{~m}\right)$ for the global loudspeaker method. Moreover, the loudspeaker (Fig. 1) has its particular coordinate system $\mathbf{X}^{\prime}, \mathbf{Y}^{\prime}$ and $\mathbf{Z}^{\prime}$ (or $\mathbf{r}^{\prime}, \boldsymbol{\theta}^{\prime}$ and $\boldsymbol{\varphi}^{\prime}$ that must be considered because the directivity characteristics of the source are referred to these coordinates. In practice, the imposition of the incidence angle $\theta_{\alpha}$ limits the loudspeaker position to a cone surface with a $45^{\circ}$ opening whose vertex is located at the origin "o" (Fig. 2). In Fig. 1, the points 1-4 refer to the façade corners, the point $P(x, y)$ corresponds to a generic point on the façade with coordinates $(x, y)$ and 0 is the point on the sample closest from the loudspeaker, whose position is variable. If the sound emitter is placed on the ground, the angle $\varphi_{\alpha}$ normally varies between the value $\varphi_{\alpha}=-90^{\circ}$ (when the loudspeaker is just below the façade) and the value $\varphi_{\alpha}=-$ a $\sin \left[\Delta y /\left(2 z_{\alpha}\right)\right]$ (when the loudspeaker is on the ground at the same height as the bottom of the façade). Nevertheless, if the sound source rises above the ground via a support element like a tripod, it can reach the position $\varphi_{\alpha}=0^{\circ}$. For those discussions where specific dimensions are required, this paper will use the façade dimensions of $\Delta x \times \Delta y=4 \mathrm{~m} \times 3 \mathrm{~m}$ (here, taken to be the standard dimensions) that can be considered typical sizes for residential buildings, although the study will also be extended to the generic case. Thus, in this standard façade, the maximum

\footnotetext{
${ }^{1}$ For compatibility with the common nomenclature for spherical coordinates, some parameter names in the ISO 140-5 have been changed. So, here $r_{\alpha} \equiv r$ ( $\mathrm{r}$ as in the standard) and $z_{\alpha} \equiv d$ ( $\mathrm{d}$ as in the standard).
} 
achievable angle is $\varphi_{\alpha}=-17.5^{\circ}$, if the loudspeaker is placed on the ground.

\subsection{Maximum direct sound pressure level differences due to spherical divergence on the façade}

The initial cause of direct sound field unevenness within the façade is spherical divergence; that is, direct sound levels decrease with distance. To determine the attenuation by distance of acoustic levels within the façade, it is very important to identify the closest and farthest points from the source within the surface. This geometrical problem is not trivial and has been extensively studied in Appendix A. As stated there, points 0 and 1 are respectively the closest and farthest ones to the sound source, when the loudspeaker is located in the fourth quadrant, facing the façade (see Fig. 1 or Fig. A1). If the loudspeaker is ideally omnidirectional, then the maximum direct sound level occurs at the point within the façade nearest from the source, point 0 , as was stated in Appendix A (and whose coordinates varies with the loudspeaker position); additionally, the minimum direct sound level will occur at the farthest point, which is always the top left corner, or point 1 . Additionally, the distance " $r$ " from the loudspeaker to the generic point $P(x, y)$ within the façade (depicted in Fig. 1) vs. the source position $\left(r_{\alpha}, \varphi_{\alpha}\right)$ is obtained in Appendix B. Therefore, using (A.1), (A.3), and (B.1), and considering that the horizontal loudspeaker projection lies outside the façade (more typical for small specimens), the ratio between the longest and shortest distances from the source within the façade is given by the following expression:

$r_{1}^{2}-2 z_{\alpha}^{2}+x_{1}^{2}+y_{1}^{2}-2 z_{\alpha}\left(x_{1} \cos \varphi_{\alpha}+y_{1} \sin \varphi_{\alpha}\right)$

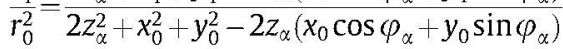

$=\left\{\begin{array}{l}\frac{8 z_{\alpha}^{2}+\Delta x^{2}+\Delta y^{2}-4 z_{\alpha}\left(-\Delta x \cos \varphi_{\alpha}+\Delta y \sin \varphi_{\alpha}\right)}{8 z_{\alpha}^{2}+\Delta y^{2}-4 z_{\alpha}^{2} \cos ^{2} \varphi_{\alpha}+4 z_{\alpha} \Delta y \sin \varphi_{\alpha}}-90^{\circ}<\varphi_{\alpha}<\varphi_{\alpha \mathrm{A}}=-\mathrm{a} \cos \frac{\Delta x / 2}{z_{\alpha}} \\ \frac{8 z_{\alpha}^{2}+\Delta x^{2}+\Delta y^{2}-4 z_{\alpha}\left(-\Delta x \cos \varphi_{\alpha}+\Delta y \sin \varphi_{\alpha}\right)}{8 z_{\alpha}^{2}+\Delta x^{2}+\Delta y^{2}-4 z_{\alpha}\left(\Delta x \cos \varphi_{\alpha}-\Delta y \sin \varphi_{\alpha}\right)} \varphi_{\alpha A}<\varphi_{\alpha}<\varphi_{\alpha B}=-a \sin \frac{\Delta y / 2}{z_{\alpha}} \\ \frac{8 z_{\alpha}^{2}+\Delta x^{2}+\Delta y^{2}-4 z_{\alpha}\left(-\Delta x \cos \varphi_{\alpha}+\Delta y \sin \varphi_{\alpha}\right)}{8 z_{\alpha}^{2}+\Delta x^{2}-4 z_{\alpha}^{2} \sin ^{2} \varphi_{\alpha}-4 z_{\alpha} \Delta x \cos \varphi_{\alpha}}-a \sin \frac{\Delta y / 2}{z_{\alpha}}=\varphi_{\alpha B}<\varphi_{\alpha}<0 .\end{array}\right.$

The expression (1) particularised to the standard façade dimensions of $4 \mathrm{~m} \times 3 \mathrm{~m}$ and with the loudspeaker at a distance $z_{\alpha}=5 \mathrm{~m}$ from the surface will be:

$$
\frac{r_{1}^{2}}{r_{0}^{2}}= \begin{cases}\frac{225+80 \cos \varphi_{\alpha}-60 \sin \varphi_{\alpha}}{209-100 \cos ^{2} \varphi_{\alpha}+60 \sin \varphi_{\alpha \alpha}} & -90^{\circ}<\varphi_{\alpha}<\varphi_{\alpha A}=-66.4^{\circ} \\ \frac{45+16 \cos \varphi_{\alpha}-12 \sin \varphi_{\alpha}}{45-16 \cos \varphi_{\alpha}+12 \sin \varphi_{\alpha}} & \varphi_{\alpha \mathrm{A}}<\varphi_{\alpha}<\varphi_{\alpha \mathrm{B}}=-17.5^{\circ} \\ \frac{225+80 \cos \varphi_{\alpha}-60 \sin \varphi_{\alpha}}{216-100 \sin ^{2} \varphi_{\alpha}-80 \cos \varphi_{\alpha}} & -17.5^{\circ}=\varphi_{\alpha \mathrm{B}}<\varphi_{\alpha}<0^{\circ} .\end{cases}
$$

For façades with generic dimensions $\Delta x \times \Delta y$, the maximum difference in distance given by (1) marks the greatest difference in direct sound pressure levels within the sample for ideal omnidirectional sources, in such a way that this difference depends on the angle $\varphi_{\alpha}$ of the emitter. This angle can be set at the least favourable angle $\varphi_{\alpha-}$ as the one that maximises the value of spherical divergence and, therefore, produces the maximum direct sound pressure level variations on the façade. Analogously, the most favourable position $\varphi_{\alpha+}$ can be also defined as that which minimises the level variations. By examining Figs. A1 and A2 in Appendix A, it is easy to determine that the least favourable angle corresponds to the one in which the projection of the loudspeaker on the $X Y$ plane is on the extension of the diagonal connecting the façade corners " 1 " and " 3 ". In this position, the corner " 1 " corresponds to the maximum distance from the source and the point " 0 " to the minimum one. On the other hand, the most favourable location to place the loudspeaker, $\varphi_{\alpha+}$, depends on the façade aspect ratio $\Delta x / \Delta y$. If the façade is a landscape $(\Delta x \mid \Delta y>1)$, then the most favourable point just lies below the centre of the façade. If the façade is vertical $(\Delta x / \Delta y<1)$, then the most favourable point will be on the horizontal of the façade centre. The latter is true because the function (1), which provides the maximum spherical divergence on the façade, presents only an absolute maximum in the interval $\left(-90^{\circ}<\right.$ $\varphi_{\alpha}<0^{\circ}$ ), corresponding to the least favourable point $\varphi_{\alpha-}$; therefore, this function has two relative minima in the variation range of $-90<\varphi_{\alpha}<0^{\circ}$. Thus, it is easily demonstrable that the lower of the two minima corresponds to $\varphi_{\alpha}=-90^{\circ}$ if $\Delta x / \Delta y>1$ and $\varphi_{\alpha}=0^{\circ}$ if $\Delta x / \Delta y<1$. Consequently, the above discussion can be summarised in the following:

$$
\begin{gathered}
\varphi_{\alpha-}=\left\{\begin{array}{ll}
-90^{\circ} \text { if } \Delta x / \Delta y>1 & \text { most favourable loudspeaker position } \\
0^{\circ} \text { if } \Delta x / \Delta y>1 &
\end{array}\right\} \\
\varphi_{\alpha-}=-a \tan (\Delta X / \Delta y) \\
-90^{\circ}<\varphi_{\alpha}<0^{\circ}
\end{gathered}
$$

Expression (3) particularised to the standard façade of $4 \mathrm{~m} \times 3 \mathrm{~m}$ will be as follows:

$$
\left.\begin{array}{l}
\varphi_{\alpha+}=-90^{\circ} \text { most favourable loudspeaker position } \\
\varphi_{\alpha-}=-37^{\circ} \text { least favourable loudspeaker position }
\end{array}\right\}-90<\varphi_{\alpha}<0 .
$$

For the standard surface of $4 \mathrm{~m} \times 3 \mathrm{~m}$, the extreme angular values from (4) substituted in (2) provide the maximum level differences for the most and least favourable angles, respectively:

$$
\frac{r_{1}}{r_{0}}[\mathrm{~dB}]=10 \log \frac{r_{1}^{2}}{r_{0}^{2}}
$$

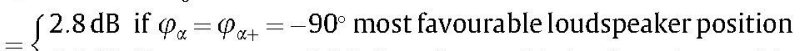

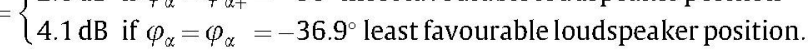

Fig. 3 shows both the most and least favourable loudspeaker positions, $\varphi_{\alpha+}$ and $\varphi_{\alpha-}$, respectively, for the façade with dimensions $4 \mathrm{~m} \times 3 \mathrm{~m}$. For the same sample, in Fig. 4 , the maximum direct sound pressure differences due to distance are shown as well as the minimum and maximum values reached for the most and least favourable loudspeaker positions ( $\varphi_{\alpha_{+}}$and $\varphi_{\alpha_{-}}$, respectively).

As shown in (5) and Fig. 4, our standard façade of $4 \mathrm{~m} \times 3 \mathrm{~m}$ (with the loudspeaker at $z_{\alpha}=5 \mathrm{~m}$ from the wall) meets the maximum level deviation permitted by ISO $140-5$, whereby if the largest façade dimension is less than $5 \mathrm{~m}$, then the maximum allowable level difference on the façade can be $\triangle \mathrm{SPL}=5 \mathrm{~dB}$. This consideration will be effective whenever the sound source is omnidirectional (difficult in practice), that is, the only direct sound

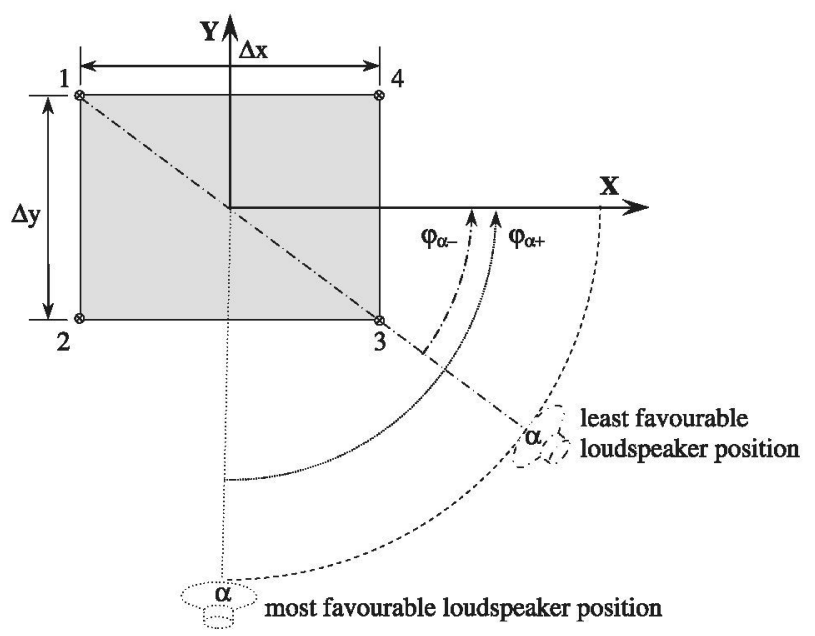

Fig. 3. Most favourable $\varphi_{\alpha+}$ and least favourable $\varphi_{\alpha-}$ loudspeaker positions for the standard façade of $4 \mathrm{~m} \times 3 \mathrm{~m}$. The most favourable position provides the least direct sound pressure differences over the sample and corresponds to the source placed just below the façade centre. The least favourable loudspeaker position corresponds to the maximum direct sound pressure differences on the façade. In this case, the source projection on the $X Y$ plane lies on the prolongation of the façade diagonal. 


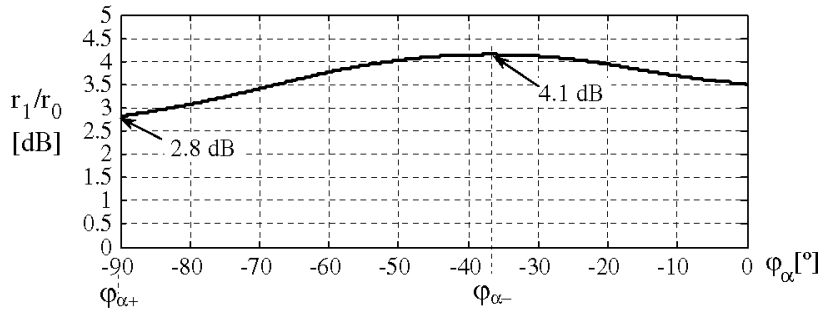

Fig. 4. Direct sound pressure level differences due to spherical divergence on the standard façade of $4 \mathrm{~m} \times 3 \mathrm{~m}$ for the most favourable loudspeaker positions $\varphi_{\alpha+}$ and the least one $\varphi_{\alpha-}$, both with $z_{\alpha}=5 \mathrm{~m}$. Only the effect of distance has been considered without taking into account air absorption, so the loudspeaker can be assumed to be ideally omnidirectional.

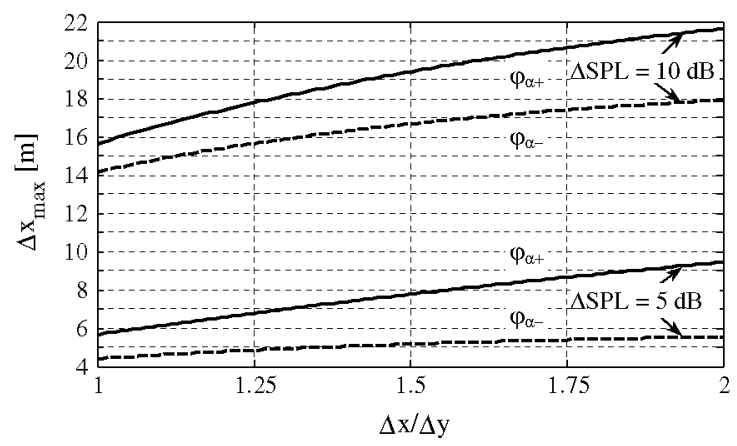

Fig. 5. Maximum horizontal dimension of the façade $\Delta x_{\max }$ that meets ISO $140-5$ either with the $\triangle \mathrm{SPL}=5 \mathrm{~dB}$ (black) or $\triangle \mathrm{SPL}=10 \mathrm{~dB}$ (grey) criteria, represented against the aspect ratio $\Delta x / \Delta y$ and for two source positions, the most and the least favourable ones, $\varphi_{\alpha+}$ and $\varphi_{\alpha-}$, respectively. In all cases, the loudspeaker has been assumed as ideally omnidirectional (so, direct level differences are caused only by spherical divergence) and located at $z_{\alpha}=5 \mathrm{~m}$.

pressure differences on the façade are caused by spherical divergence. However, although the façade of $4 \mathrm{~m} \times 3 \mathrm{~m}$ has been chosen as representative in this text, in practice, its size may be different. Given the aspect ratio $\Delta x / \Delta y$, the maximum façade dimension $\Delta x_{\max }$ for ISO $140-5$ can be easily determined using (1), either for $\Delta \mathrm{SPL}=5 \mathrm{~dB}$ (considered if $\Delta x \leqslant 5 \mathrm{~m}$ ) or $\Delta \mathrm{SPL}=10 \mathrm{~dB}$ (if $\Delta x>$ $5 \mathrm{~m}$ ), maintaining that the loudspeaker is ideally omnidirectional. In Fig. 5, the maximum façade dimension $\Delta x_{\max }$ corresponding to ISO $140-5$ is plotted against the aspect ratio $\Delta x / \Delta y$, both for the $\triangle \mathrm{SPL}=5 \mathrm{~dB}$ and the $\triangle \mathrm{SPL}=10 \mathrm{~dB}$ criteria, and in the most and least favourable positions, $\varphi_{\alpha+}$ (full line) and $\varphi_{\alpha_{-}}$(dashed line), respectively. In all cases, it has been assumed that $z_{\alpha}=5 \mathrm{~m}$ and that the loudspeaker is omnidirectional, such that acoustic levels are influenced only by spherical divergence.

Although in Fig. 5, the effects of the loudspeaker directivity are not considered, it can provide a good estimate of the maximum feasible sizes of the façade that meet the ISO standard. It can be seen that the greater aspect ratios $\Delta x / \Delta y$ (landscape) are beneficial because they allow larger façade horizontal dimensions. For example, for the standard façade here considered $(\Delta x / \Delta y=4 / 3)$, a maximum horizontal dimension of $\Delta x_{\max }=7 \mathrm{~m}$ would be allowed for the most favourable position of the source, $\varphi_{\alpha+}=-90^{\circ}$, but instead, $\Delta x_{\max }=5 \mathrm{~m}$ would be allowed for the least favourable one, $\varphi_{\alpha_{-}}=-36.9^{\circ}$.

\section{Combined effect of loudspeaker directivity and spherical divergence on acoustic levels}

At each point $P(x, y)$ on the façade, the combined effect $D_{r}$ of the distance $r$, as in expression (B.1) from Appendix B, and the loud- speaker directivity $D$ over direct acoustic levels within the façade will be given by the product of the inverse of the distance with the directivity as follows:

$D_{r}=D / r$

If the loudspeaker is ideally omnidirectional, then the directivity term $D \equiv D\left(\theta^{\prime}, \varphi^{\prime}\right)=1$ and $D_{r}=1 / r$, such that the maximum differences of direct sound pressure levels on the façade will be those discussed in the previous section (if air absorption is excluded). However, in practice, to perform the building insulation tests following the ISO standard, the source used will usually not be omnidirectional; thus, it seems necessary to study the combined effects of spherical divergence and directivity using (6).

A priori, the effect of loudspeaker directivity is difficult to predict. It may be assumed that the typical directivity functions of the sound sources with a prominent front lobe could compensate for the level differences on the façade due to the distance by appropriately aiming the loudspeaker. However, this procedure is difficult to implement, and high loudspeaker directivity increases level differences on the sample, preventing the sound source from fulfilling the ISO standard.

To determine the influence of loudspeaker directivity $D\left(\theta^{\prime}, \varphi^{\prime}\right)$ in the acoustic levels on the façade, it is necessary to know the relative angles of the generic façade points $P(x, y)$ with respect to the loudspeaker coordinate system, wherein the directivity functions are defined. The loudspeaker coordinate system is the orthonormal Cartesian axis system defined by $\mathbf{X}^{\prime}, \mathbf{Y}^{\prime}$ and $\mathbf{Z}^{\prime}$, as shown in Fig. 1 (or, also the orthonormal spherical coordinate system $\mathbf{r}^{\prime}, \boldsymbol{\theta}^{\prime}$ and $\varphi^{\prime}$, not shown in Fig. 1 for simplicity). Thus, it seems crucial to determine the angular coordinates of each façade point in the loudspeaker coordinate system, that is, the elevation and azimuth angles, $\theta^{\prime}(x, y)$ and $\varphi^{\prime}(x, y)$, respectively. This discussion is developed in Appendix $C$, and the following summarises the results obtained for the coordinates $(x, y)$ of each façade point, expressed in the loudspeaker coordinate system:

$$
\begin{aligned}
r^{\prime} \equiv r & =\sqrt{x^{2}+y^{2}+2 z_{\alpha}^{2}-z_{\alpha}\left(x \cos \varphi_{\alpha}+y \sin \varphi_{\alpha}\right)} \\
\cos \theta^{\prime} & =\frac{2 z_{\alpha}-x \cos \varphi_{\alpha}-y \sin \varphi_{\alpha}}{\sqrt{2 x^{2}+2 y^{2}+4 z_{\alpha}^{2}-4 z_{\alpha}\left(x \cos \varphi_{\alpha}+y \sin \varphi_{\alpha}\right)}} \\
\tan \varphi^{\prime} & =\frac{\sin \varphi_{\alpha} \cos \varphi_{\alpha}}{\sqrt{2}}-\frac{y\left(1+\cos ^{2} \varphi_{\alpha}\right)}{x \sqrt{2}}
\end{aligned}
$$

Next, this section will discuss the influence of loudspeaker directivity on direct sound levels over the façade.

\subsection{Maximum angular coverage of the façade with respect to the loudspeaker}

Over the possible values of angle $\varphi_{\alpha}$ between $-90^{\circ}$ and $0^{\circ}$, the maximum elevation angle $\theta^{\prime}$ with which the source "sees" the façade is determined by the four surface corners (Fig. 1), that is, the points "1" (top left), "2" (bottom left), "3" (bottom right) and "4" (top right). At each loudspeaker angle $\varphi_{\alpha}$, the maximum elevation angle $\theta^{\prime}$ corresponding to the four corners, $\theta_{1}^{\prime}, \theta_{2}^{\prime}, \theta_{3}^{\prime}$ and $\theta_{4}^{\prime}$, will determine the maximum angular coverage of the façade with respect to the loudspeaker. Therefore, if the loudspeaker aims towards the façade centre, then the maximum angular coverage needed for the loudspeaker (measured at both sides of the axis, as is usual in loudspeakers) will be twice the maximum $\theta^{\prime}$ chosen among the four corners $\theta_{1}^{\prime}, \theta_{2}^{\prime}, \theta_{3}^{\prime}$ and $\theta_{4}^{\prime}$ :

$\theta_{\max }^{\prime}=2 \cdot \max \left(\theta_{1}^{\prime}, \theta_{2}^{\prime}, \theta_{3}^{\prime}, \theta_{4}^{\prime}\right)$,

with $\theta_{1}^{\prime}, \theta_{2}^{\prime}, \theta_{3}^{\prime}$ and $\theta_{4}^{\prime}$ the elevation angles of the four façade corners in the loudspeaker coordinate system. 


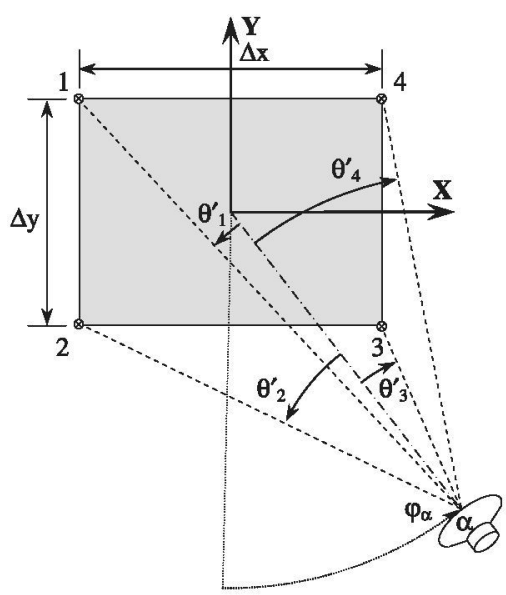

Fig. 6. Maximum coverage angles of the façade with respect to the loudspeaker that are determined by the elevation angles $\theta_{1}^{\prime}, \theta_{2}^{\prime}, \theta_{3}^{\prime}, \theta_{4}^{\prime}$ of the façade corners in the loudspeaker reference system. When the sound source aims towards the façade centre, the maximum loudspeaker coverage, considered at both axis sides, which covers the entire façade, will be considered to be twice the maximum elevation angle chosen among the four façade corners, $\theta_{1}^{\prime}, \theta_{2}^{\prime}, \theta_{3}^{\prime}$ and $\theta_{4}^{\prime}$.

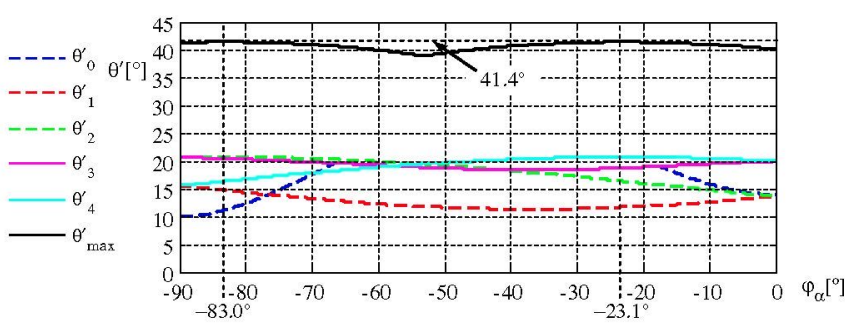

Fig. 7. The elevation angles of the four corners, $\theta_{1}^{\prime}, \theta_{2}^{\prime}, \theta_{3}^{\prime}$ and $\theta_{4}^{\prime}$, are plotted in the loudspeaker reference system as a function of $\varphi_{\alpha}$ for the standard façade of $4 \mathrm{~m} \times 3 \mathrm{~m}\left(z_{\alpha}=5 \mathrm{~m}\right)$. The maximum coverage angle $\theta_{\max }^{\prime}$ from (7) is also shown. $\theta_{\max }^{\prime}$ is the angle at which the loudspeaker "sees" the façade. The elevation angle $\theta_{0}^{\prime}$ of the closest point from the sound source in the loudspeaker reference system is represented as well.

Fig. 6 displays the elevation angles of the four façade corners, $\theta_{1}^{\prime}$, $\theta_{2}^{\prime}, \theta_{3}^{\prime}$ and $\theta_{4}^{\prime}$, in the loudspeaker reference system and, therefore, the angular coverage with which the sound source "sees" the façade. In Fig. 7, these four elevation angles of the selected standard façade corners (size $4 \mathrm{~m} \times 3 \mathrm{~m}$ and with $z_{\alpha}=5 \mathrm{~m}$ ) are plotted as a function of $\varphi_{\alpha}$, in the loudspeaker coordinate system. Also, the maximum coverage angle $\theta_{\max }^{\prime}$, with which the loudspeaker "sees" the façade, as in (7) is represented. As can be visualised in Fig. 7, $\theta_{\max }^{\prime}$ reaches its maximum value of $41.4^{\circ}$ at $\varphi_{\alpha}=83.0^{\circ}$ and $\varphi_{\alpha}=23.1^{\circ}$. Therefore, according to Fig. 7, for the case of the $4 \mathrm{~m} \times 3 \mathrm{~m}$ dimensions, it is sufficient to consider the sound source directivity up to approximately $40^{\circ}$ when studying the loudspeaker effects on the direct sound levels on the façade.

\subsection{Maximum façade dimensions for pistons mounted in infinite baffle}

Thus far, only the influence of distance on direct acoustic levels on the façade has been studied. Excluding the air absorption influence, the conclusions of Section 2 on the maximum façade dimensions that meet the ISO standard can be easily generalised to all sound sources, provided that they are omnidirectional. The omnidirectionality condition is approximately fulfilled at a low frequency for conventional box-type sound sources or, more specifically, for $k a \ll 1$, where " $k$ " is the wave number and " $a$ " is some characteristic dimension of the sound emitter, such as its radius. Section 3.1 has also studied the maximum angular coverage with which the loudspeaker "sees" the façade and that determines the angles at which the loudspeaker directivity influences the direct acoustic levels on the surface. Thus, given a certain façade size, the loudspeaker directivity should be "controlled" in the angle $\theta_{\max }^{\prime}$ as defined in (7) in order to meet the ISO standard. If the sound source directivity function $D\left(\theta^{\prime}, \varphi^{\prime}\right)$ is known, it can be easily combined with the distance to calculate the directivity to distance ratio $D_{r}(6)$, using C.4, C.5, and C.18 from Appendix C. However, it is difficult to advance what should be the shape of the loudspeaker directivity function inside the coverage $\theta_{\max }^{\prime}$ to meet the required uniformity for the direct acoustic field on the façade. Given certain directivity functions of the loudspeaker, it would be very helpful to know what will be the maximum façade size that can fulfil the ISO standard requirements. Although loudspeakers may have all types of directivity shapes, normally the ones used for the ISO 140-5 measurements contain acoustic sources in one- or two-way configuration, either coaxially or line-mounted, and they often have a single front radiation lobe, except for the crossover frequencies in the two-way systems. In this section, the effect on direct sound field of the piston directivity function, according to its known expression [4], will be calculated and discussed.

The directivity function for the piston mounted in an infinite baffle is:

$D\left(\theta^{\prime}\right)=\frac{2 J_{1}\left(k a \cdot \sin \theta^{\prime}\right)}{k a \cdot \sin \theta^{\prime}}$

where $J_{1}(\cdot)$ is the Bessel function of the first kind and first order and " $a$ " is the piston radius.

Fig. 8 shows the maximum horizontal dimension $\Delta x_{\max }$ of the façade that meets ISO $140-5$, either with the $\triangle S P L=5 \mathrm{~dB}$ (in black colour) or $\triangle \mathrm{SPL}=10 \mathrm{~dB}$ (grey colour) criteria, represented as a function of the $-6 \mathrm{~dB}$ coverage angle $\theta_{L(-6 \mathrm{~dB})}^{\prime}$, for a piston radiating in infinite baffle that fulfils ( 8 ). The curves have been obtained by calculating the direct sound levels on façades of varying dimensions, considering the sound source as a piston with different coverage angles $\theta_{L(-6 \mathrm{~dB})}^{\prime}$ according to the value of the "ka" factor, as it appears in the formula (8), and combined with the distance (C.4) and the elevation angle (C.5) formulas. The piston is located at a distance $z_{\alpha}=5 \mathrm{~m}$ from the façade, and it is aimed towards its centre. The graphs represent both the most favourable source position $\varphi_{\alpha^{+}}$(full line) and the least favourable position $\varphi_{\alpha_{-}}$(dashed line). The end of the curves towards the right corresponds to the maximum piston coverage $\theta_{L(-6 \mathrm{~dB})}^{\prime}$ that is able to meet the ISO standard. On the right scale of each graph, the maximum horizontal dimensions $\Delta x_{\max }$ represent the case of the ideal omnidirectional loudspeaker for the same results as shown in Fig. 5. That is, for the nearby omnidirectional sources with directivity losses less than $6 \mathrm{~dB}$, the maximum horizontal dimensions $\Delta x_{\max }$ that meet the ISO standard would be as if only spherical divergence is considered, and these dimensions are indicated on the right side of the Fig. 8 graphs, and more precisely in Fig. 5. The four graphs correspond to four façade aspect ratios: $\Delta x|\Delta y=1, \Delta x| \Delta y=4 / 3, \Delta x \mid$ $\Delta y=3 / 2$ and $\Delta x / \Delta y=2$.

Some conclusions can be drawn from Fig. 8. First, at least for the piston pointing to the centre of the façade, it seems that directive sources always work worse than ideal omnidirectional ones, i.e., their façade coverage with the ISO standard criteria is lower. Directivity functions may be hypothesised to better compensate for the higher levels in the points closest to the source, but this compensation is not easily allowable, at least for lobe-type directivity patterns. To significantly improve the acoustic level differences with the source aimed at a position other than the façade centre, by trying to compensate for distance with directivity, is neither expected Moreover, Fig. 8 gives an outline of the maximum façade size that 

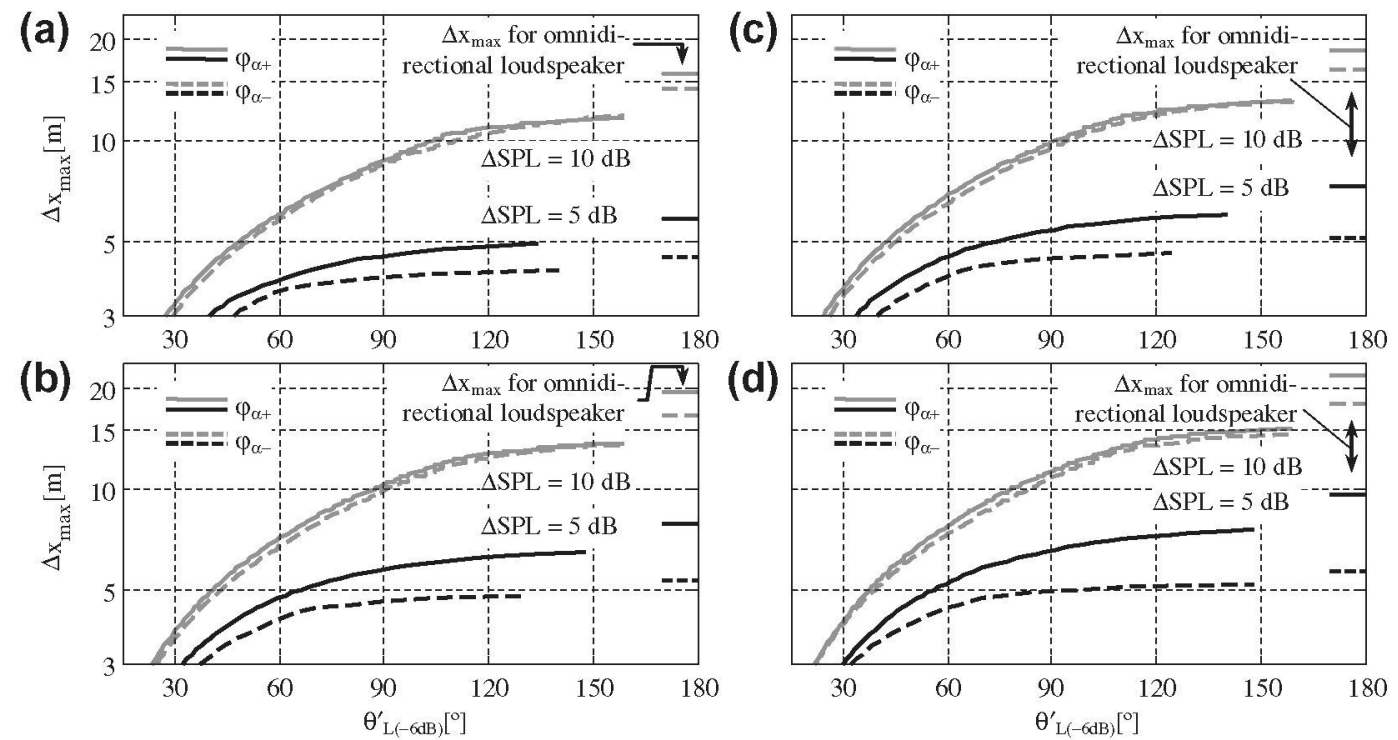

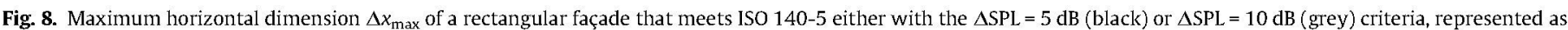

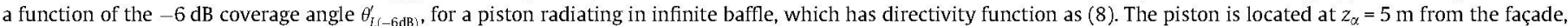

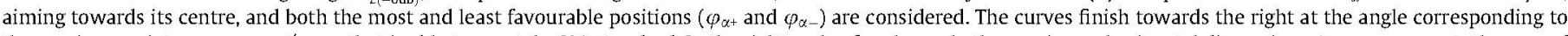

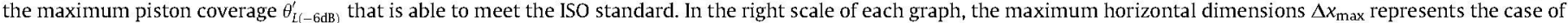

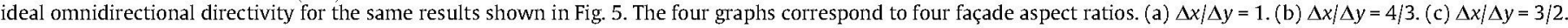
(d) $\Delta x / \Delta y=2$.

meets the ISO standard if the source directivity is known, characterised by its $-6 \mathrm{~dB}$ coverage angle $\theta_{L(-6 \mathrm{~dB})}^{\prime}$. However, such correspondence can only be applied to sources with regular directivity functions such as single lobe shapes and is lost for more complicated patterns.

\section{Estimation of direct acoustic levels on façades produced by typical loudspeaker configurations}

In this section, direct acoustic levels on a façade according to ISO 140-5 produced by a series of loudspeakers for which the electroacoustic data are known are simulated and discussed. Level simulations were performed with the software program EASE 4.3 [5]. The electroacoustic data of the loudspeakers, especially the directivity patterns, were either simulated or came from laboratory measurements. The sound emitters chosen for the simulations in this section correspond to the radiating configurations commonly used in the acoustic insulation tests, as proposed in the ISO 140-5.

In the real world, the loudspeakers that are frequently used for façade measurements according to ISO 140-5 respond to radiation models that are easily classifiable as either one-way or two-way sound sources with crossover frequencies $f_{c}$ located approximately in the centre of the audio spectrum and as using either "coaxial" or "in line" settings. The acoustic emitters constituting the aforementioned loudspeaker systems can be more or less matched (at least in low frequency, namely, the loudspeaker "piston range") to the radiation pattern of a piston mounted in infinite baffle that follows (8), although in the market, there are loudspeaker box systems with horn-type tweeters that have radiation patterns that are different from that of the piston.

The acoustic sources simulated in this section have sound radiators modelled by different-sized pistons, represented by the radius " $a$ ". There are two fundamental parameters that affect the radiation pattern of any of the studied models: the loudspeaker size vs. the wavelength through the factor " $k a$ ", and the crossover frequency $f_{c}$ for the two-way systems. Listed below are the modelled loudspeaker configurations (see Fig. 9): (a) One-way (radius $a_{1}=10 \mathrm{~cm}$ ).

(b) Two-way in "coaxial" configuration (radius $a_{1}=10 \mathrm{~cm}$ and $\left.a_{2}=2 \mathrm{~cm}\right), f_{c}=1 \mathrm{kHz}$.

(c) Two-way "in line" configuration (radius $a_{1}=10 \mathrm{~cm}$ and $\left.a_{2}=2 \mathrm{~cm}\right), f_{c}=1 \mathrm{kHz}$.

(d) Two-way commercial loudspeaker Meyer Sound UP Junior ( $8^{\prime \prime}$ cone $+2^{\prime \prime}$ horn-type tweeter)

(e) Dodecahedron loudspeaker using sources with radius $6 \mathrm{~cm}$.

The directivity of the sources for all of the models except (d) was modelled using the piston expression (8), to which was added the back diffraction of the loudspeaker box, although this effect does not influence the façade levels. The radiation from the constitutive sources was combined using the "EaseSpkr" software, included in EASE, to obtain the joint directivity patterns [6,7]. As a special case, data for the loudspeaker (d) come from electroacoustic characterisation measurements performed by the authors in an anechoic room on the physical loudspeaker. Using these measurements, the loudspeaker (d) was also modelled in "EaseSpkr" for EASE simulations.

Two piston sizes were modelled for the simulations, $a_{1}=10 \mathrm{~cm}$ and $a_{2}=2 \mathrm{~cm}$, which represent typical bass and treble units. A crossover frequency $f_{C}=1 \mathrm{kHz}$ was also set for the two-way systems. The dodecahedron loudspeaker was modelled by pistons of a radius $6 \mathrm{~cm}$, obtaining the joint directivity patterns that proved to be very similar to real dodecahedron patterns measured in the laboratory by the authors. In Fig. 10 , the $-6 \mathrm{~dB}$ horizontal and vertical coverage angles $\theta_{L(-6 \mathrm{~dB})}^{\prime}$ are represented as a function of the frequency of the five loudspeakers considered.

Using EASE, direct sound pressure level calculations were performed in the third octave bands at the $100 \mathrm{~Hz}-5 \mathrm{kHz}$ range over a $22 \mathrm{~m} \times 22 \mathrm{~m}$ façade with $5 \mathrm{~cm}$ accuracy both horizontally and vertically. For each loudspeaker, five positions were contemplated: one for the most favourable $\varphi_{\alpha_{-}}=-90^{\circ}$ and four for the least favourable, $\varphi_{\alpha-}=-45^{\circ},-36.9^{\circ},-33.7^{\circ}$ and $-26.6^{\circ}$, depending on the façade aspect ratio considered, $\Delta x / \Delta y=1,4 / 3,3 / 2$ and 2 . In all cases, the sound source is placed horizontally (coordinate axes $\mathbf{X}^{\prime}$ and $\mathbf{X}$ are coplanar; see Appendix $C$ ) at a distance $z_{\alpha}=5 \mathrm{~m}$ from 

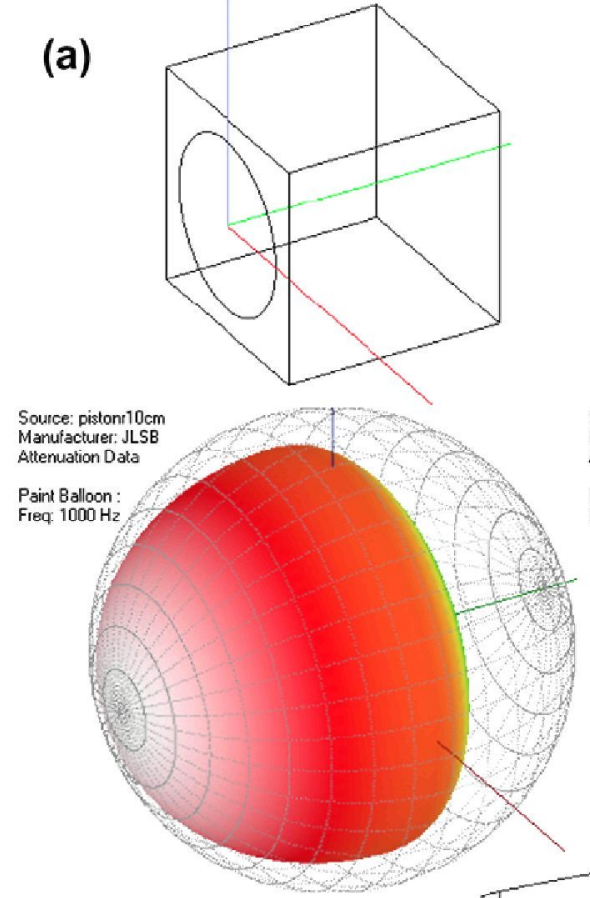

(d)
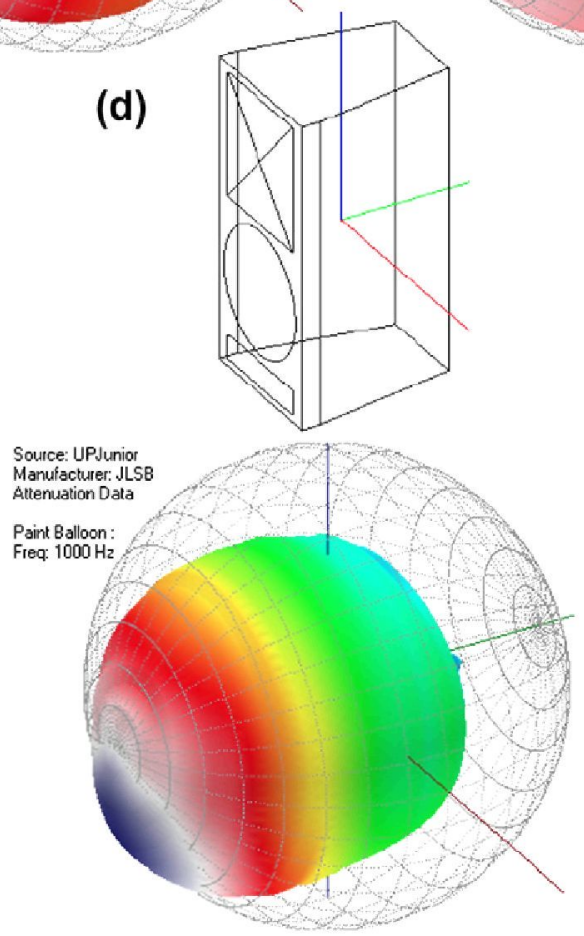

(b)

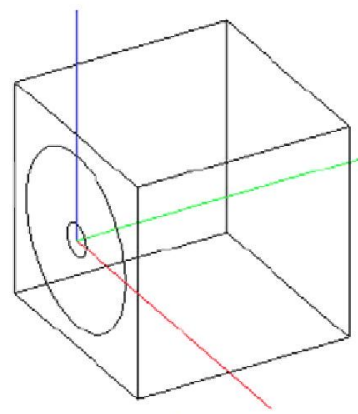

(c)
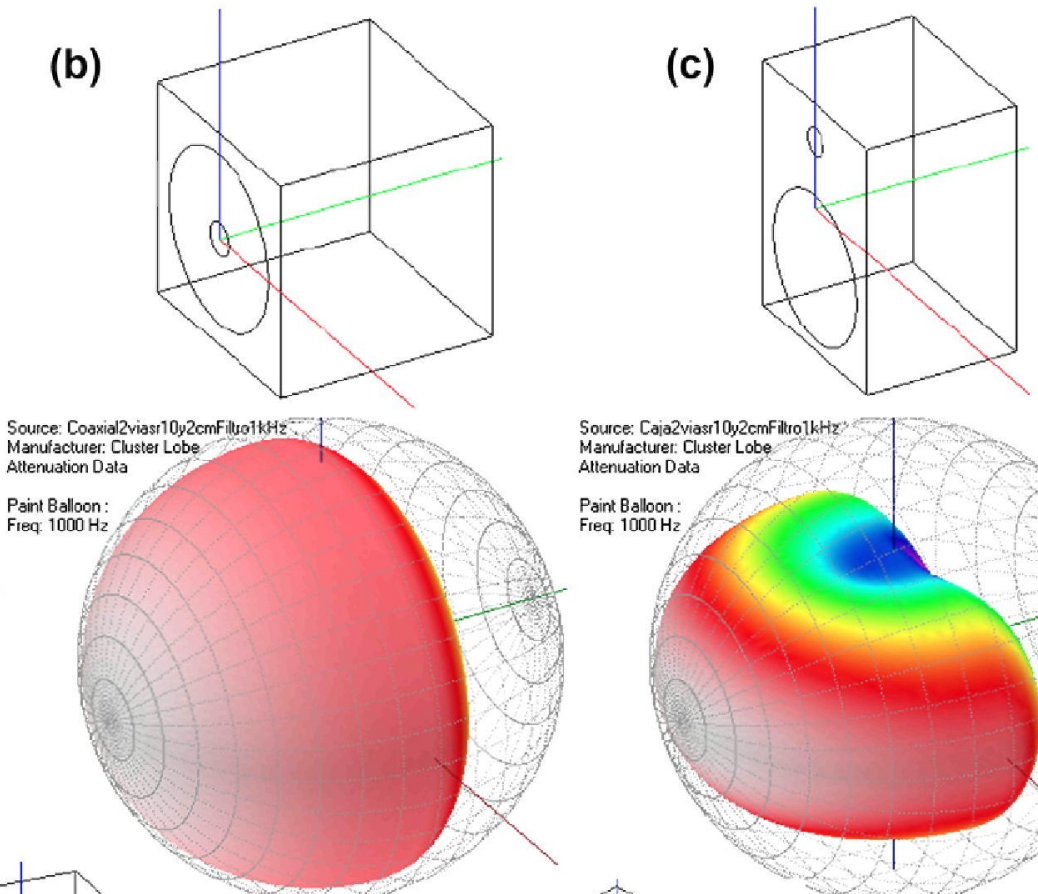
Source: Caja2viasi 10y2cmfilitrolkHz
Manulacturen: Cluster Lobe Altenuation Data

Paint Balloon: Paint Balloon:
Freq $1000 \mathrm{~Hz}$

(e)
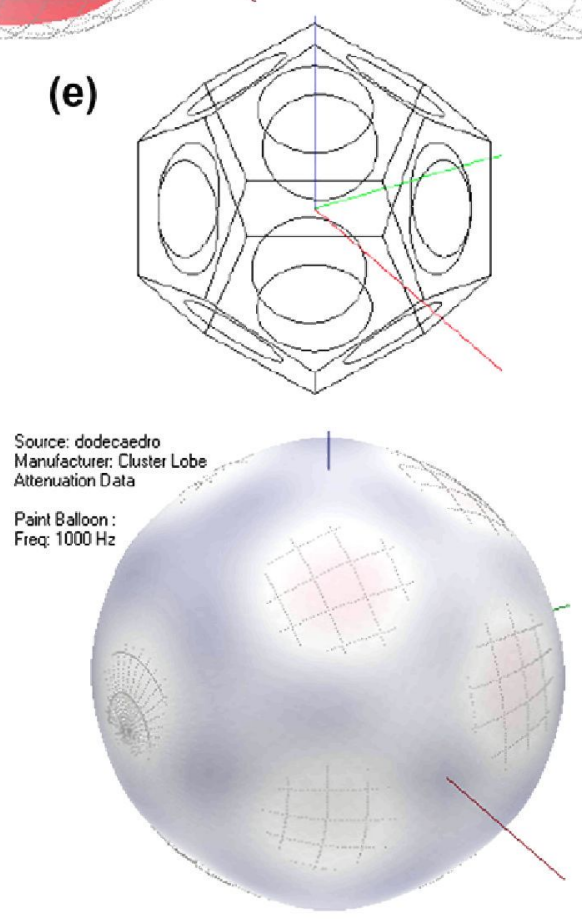

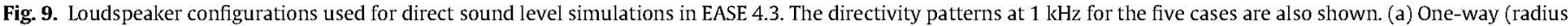

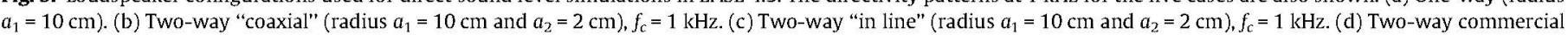
model with horn-type tweeter. (e) Dodecahedron loudspeaker with sources of radius $6 \mathrm{~cm}$.

the façade and aimed towards its centre. With the initially obtained levels, the maximum horizontal dimension $\Delta x_{\max }$ meeting ISO 140-5 was calculated for the four aspect ratios, $\Delta x / \Delta y=1,4$ / $3,3 / 2$ and 2 , and for the two level difference criteria, $\Delta \mathrm{SPL}=5 \mathrm{~dB}$ and $\Delta \mathrm{SPL}=10 \mathrm{~dB}$. In addition, we also identified in which octave band the worst case occurred.

In Fig. 11, the highest sound pressure level differences $(\triangle S P L)$ obtained after EASE predictions are represented on the standard façade with $\Delta x \times \Delta y=4 \mathrm{~m} \times 3 \mathrm{~m}$ and $z_{\alpha}=5 \mathrm{~m}$, measured in third octave bands and represented as a function of frequency for the five loudspeakers along with the "sphere" (omnidirectional case). The sound source was located both at the most and at the least favourable positions, $\varphi_{\alpha+}=-90^{\circ}$ and $\varphi_{\alpha_{-}}=-36.9^{\circ}$, respectively Fig. 12 shows an example of direct acoustic maps obtained with EASE on the standard sample and in the two proposed positions, the most and the least favourable.

In Fig. 13, the overall results for all simulations are represented. The five loudspeakers above described - (a), (b), (c), (d) and (e) have been considered. The results for these five cases (red lines) ${ }^{2}$ are compared one by one with an omnidirectional sound source generated by EASE 4.3 (called "sphere"), which is, as is shown in

\footnotetext{
${ }^{2}$ For interpretation of color in Fig. 13, the reader is referred to the web version of this article.
} 

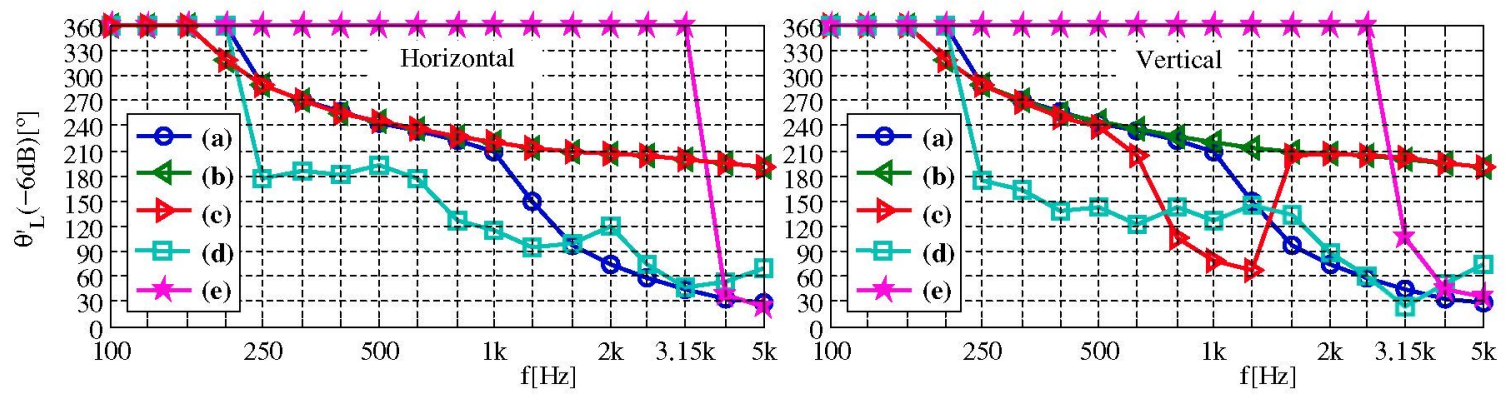

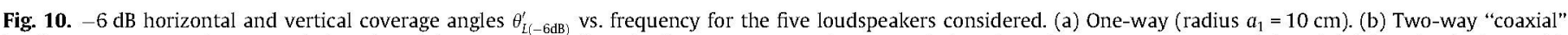

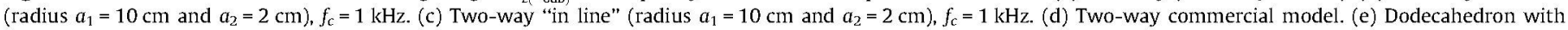
loudspeakers of radius $6 \mathrm{~cm}$.

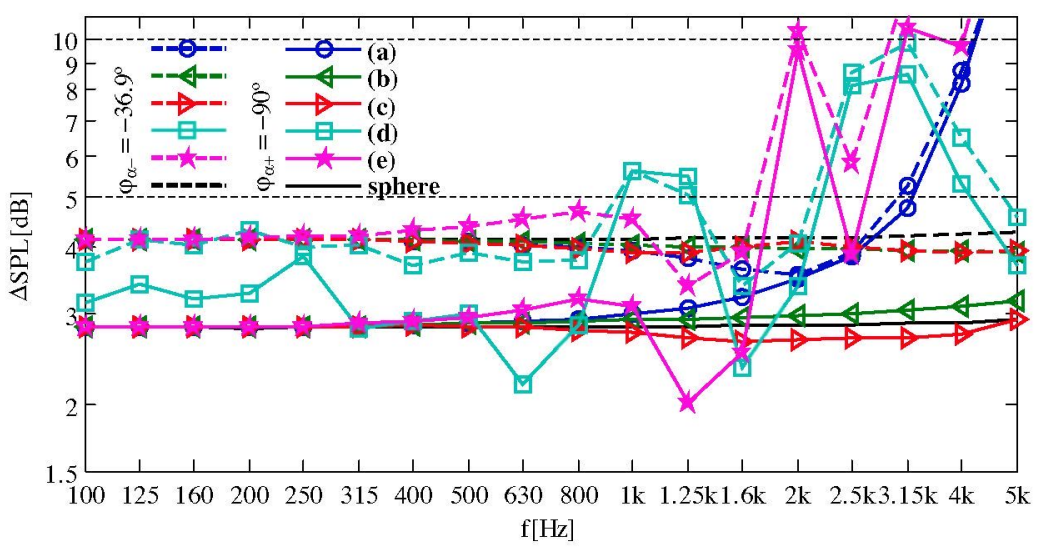

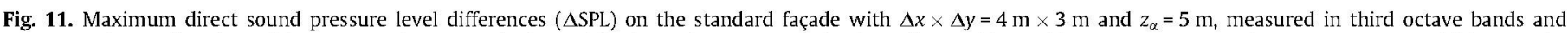

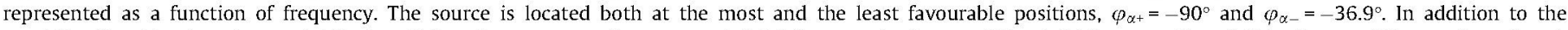

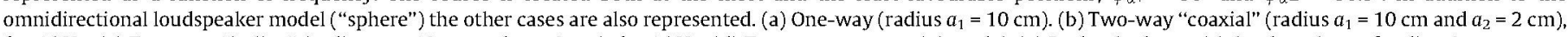
$f_{c}=1 \mathrm{kHz}$. (c) Two-way "in line" (radius $a_{1}=10 \mathrm{~cm}$ and $a_{2}=2 \mathrm{~cm}$ ), $f_{c}=1 \mathrm{kHz}$. (d) Two-way commercial model. (e) Dodecahedron with loudspeakers of radius $6 \mathrm{~cm}$.

the results (black and grey lines), the loudspeaker that achieves the least direct SPL differences on the façade and thus the largest surface dimensions. In fact, the curves corresponding to the omnidirectional loudspeaker of Fig. 13 (top and black for $\triangle \mathrm{SPL}=5 \mathrm{~dB}$ or grey for $\triangle S P L=10 \mathrm{~dB}$ ) should be identical to those shown in Fig. 5. This coincidence is not completely met because in Fig. 13, sound absorption of the air has been taken into account (for a relative humidity of $60 \%$, temperature of $20^{\circ} \mathrm{C}$ and static atmospheric pressure of $1013 \mathrm{hPa}$ ), and this effect is known to decrease levels at high frequency and long distance, slightly reducing the façade size when obtained with the EASE 4.3 simulations. In Fig. 13, the full lines correspond to the most favourable positions (always $\varphi_{\alpha^{+}}=-90^{\circ}$ for all aspect ratios $\Delta x / \Delta y$ plotted), and the dashed lines are for the least favourable ones $\left(\varphi_{\alpha_{-}}=-45^{\circ},-36.9^{\circ}\right.$, $-33.7^{\circ},-26.6^{\circ}$, depending on the façade aspect ratio $\Delta x / \Delta y$ modelled). For each loudspeaker model, the upper and lower pair of curves refer respectively to the $\triangle S P L=5 \mathrm{~dB}$ (dark red) and $\triangle \mathrm{SPL}=10 \mathrm{~dB}$ (bright red) criteria.

As an example, in Table 1 the maximum horizontal dimensions $\Delta x_{\max }[\mathrm{m}]$ extracted from Fig. 13 for the particular case of the aspect ratio $\Delta x / \Delta y=4 / 3$ are shown.

From Fig. 13, significant conclusions can be extracted. Among the selected cases, the best loudspeaker is the two-way source in coaxial arrangement, Fig. 13b, which obtains façade sizes that are even slightly larger in some cases than those obtained by the omnidirectional or "sphere" source. The two-way "in line" acoustic source arrangement, Fig. $13 c$, obtains similar results to the previous one when its sound radiation is from the most favourable position $\varphi_{\alpha^{+}}=-90^{\circ}$, but worse results for oblique radiation positions.
This finding is mainly due to the narrowing of the directivity pattern at medium frequencies (near the crossover frequency $f_{c}$ ), as can be appreciated in Fig. 9c, which penalises the loudspeaker oblique positions, although not so much when it radiates from $\varphi_{\alpha+}=-90^{\circ}$, supposedly because the polar directivity pattern matches to some extent the rectangular façade shape.

On the other hand, the one-way source, Fig. 13a, produces worse results than does the two-way one, covering smaller surfaces. This fact was predictable, as the piston directivity with radius $10 \mathrm{~cm}$ is very narrow above $2.5 \mathrm{kHz}$. The only real loudspeaker analysed, Fig. 13d, is a two-way source arranged "in line" and with a horn-type tweeter. It produces worse results than do the previous cases. A more detailed analysis of coverage maps obtained on the façade (not shown) leads to the conclusion that complex directivity patterns that are associated with horn loudspeakers, with high lobulation and different coverage angles in the horizontal and vertical planes, may be the cause of the poor performance. Finally, the dodecahedron source, Fig. 13e, produces the worst results, mainly due to excessive directivity lobulations present above $2 \mathrm{kHz}$.

Additionally, as was initially intended in this paper, some correlations between the $-6 \mathrm{~dB}$ coverage angle of loudspeakers, $\theta_{L(-6 \mathrm{~dB})}^{\prime}$, and the maximum size of the façade covered can be established, at least for loudspeakers with coverage angles meeting the condition $\theta_{L(-6 \mathrm{~dB})}^{\prime}<180^{\circ}$ (that is, excluding sound sources with very wide radiation) and directivity patterns of single-lobe shapes; these conditions discard the cases (b) two-way coaxial, because it is almost omnidirectional in the selected frequency, range and (e) dodecahedron which has high directivity lobulation from $2 \mathrm{kHz}$. On the 


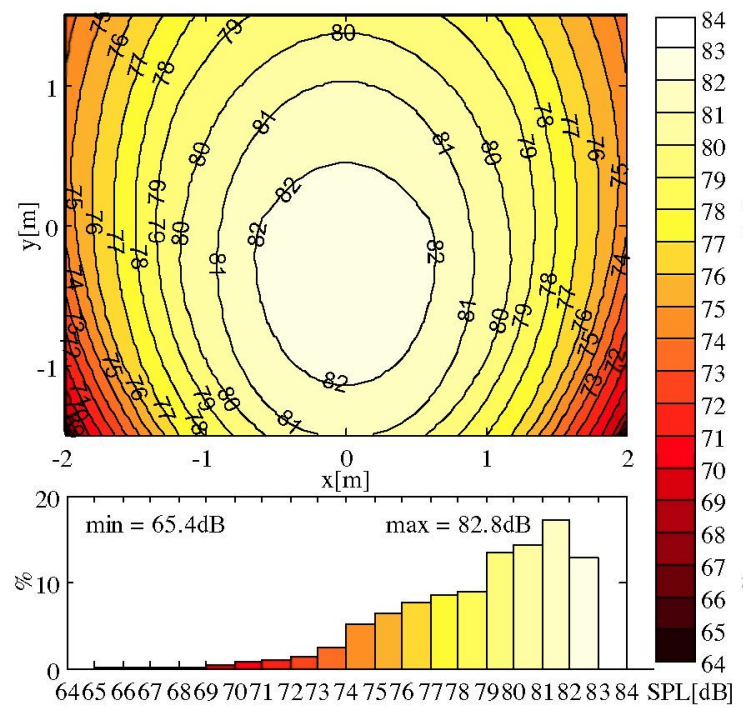

(a)

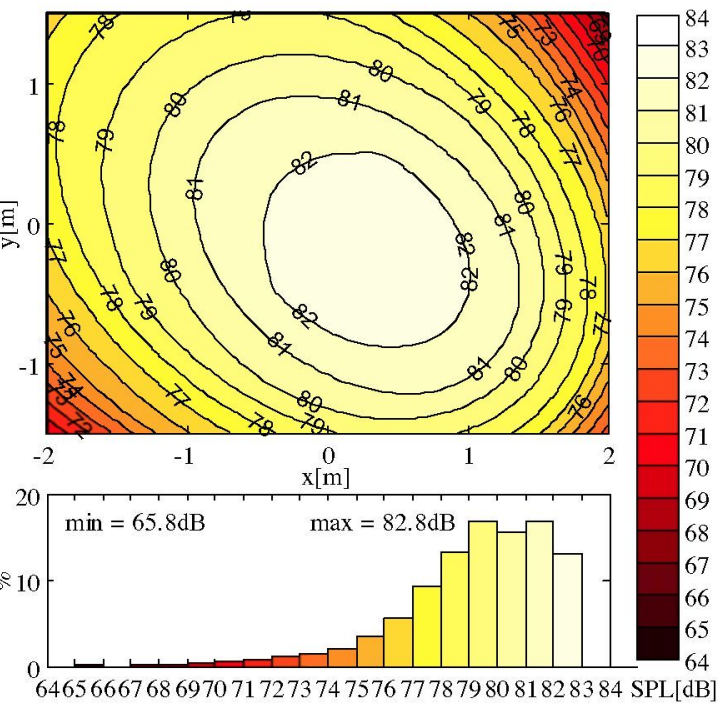

(b)

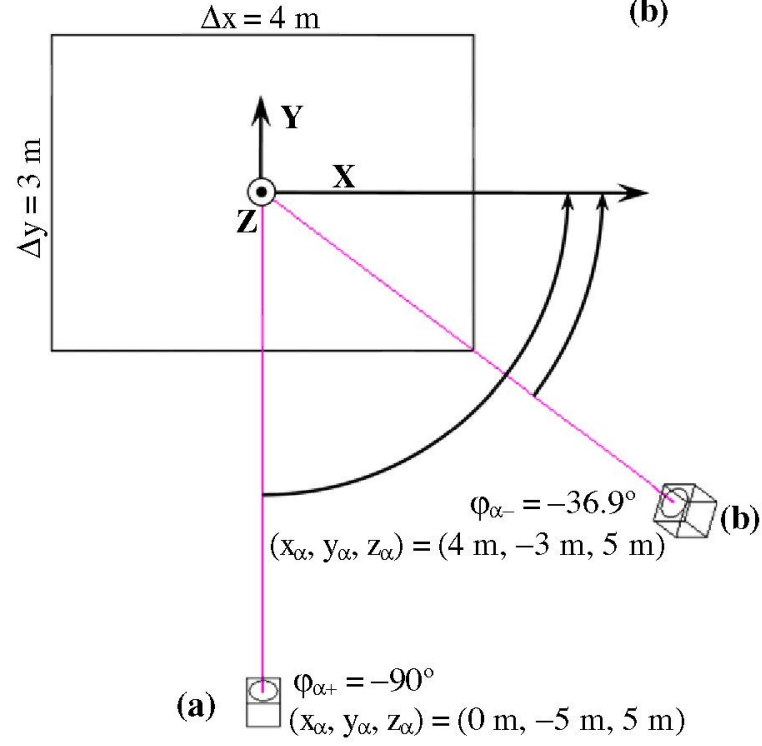

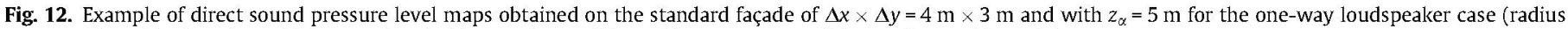
$a_{1}=10 \mathrm{~cm}$ ) and in the $5 \mathrm{kHz}$ third octave band. (a) Most favourable position $\varphi_{\alpha_{+}}=-90^{\circ}$. (b) Least favourable position $\varphi_{\alpha-}=-36.9^{\circ}$.

other hand, for example, both the one-way loudspeaker in Fig. 13a and the real loudspeaker in Fig. 13d allow similar façade sizes, at least for the maximum level difference of $\Delta \mathrm{SPL}=10 \mathrm{~dB}$. This coincidence can be attributed to its similar minimum coverage angle $\theta_{L(-6 \mathrm{~d} \mathbf{B})}^{\prime} \approx 30^{\circ}$ (Fig. 10). Moreover, the two-way "in line" loudspeaker case depicted in Fig. $13 \mathrm{c}$ allows the maximum façade dimensions of $\Delta x_{\max } \approx 5 \mathrm{~m}$ for $\Delta \mathrm{SPL}=5 \mathrm{~dB}$ and $\Delta x_{\max } \approx 10 \mathrm{~m}$ for $\triangle \mathrm{SPL}=10 \mathrm{~dB}$. In Fig. 10 , the minimum coverage angle for this two-way source is $\theta_{L(-6 \mathrm{~dB})}^{\prime} \approx 60^{\circ}$, which using Fig. 8 applied to pistons verifies approximately the same maximum dimensions of $\Delta x_{\max } \approx 5 \mathrm{~m}$ and $\Delta x_{\max } \approx 10 \mathrm{~m}$ obtained through EASE simulations. Thus, in certain cases, the coverage angle can be related to the maximum allowable surface for the ISO $140-5$ restrictions.

\subsection{Additional considerations: Effect of reflections}

Reading the ISO 140-5 standard, it seems very clear that the parameter to validate the sound source is the direct sound level on the façade. However, it is also clear that direct sound levels on the façade are modified significantly when considering acoustic reflections in the surfaces involved in the measurements [8]. At the very least, there will be wave reflections on the façade surface and on the ground, although other different walls can also affect the results. The reflection on the façade increases the sound level on the front, depending on its absorption coefficient for different frequencies. The increase in this level will not change a priori the level distribution on the façade. On the other hand, the reflection on the ground is much more harmful, producing a radical change in the level distribution over the façade, depending on the ground absorption coefficient and the distance from the sound source to the floor. This paper is not intended to comprehensively cover the effect of reflections as deep as has been done with the direct acoustic levels, but it could be sufficient to state here that reflections highly modify the acoustic level distribution on the façade. Thus, verification of the source directivity according ISO 140-5 away from a pure acoustic free-field (e.g., outdoors) may be not advisable, and the anechoic room is preferred.

In Fig. 14, the maximum sound levels differences $\Delta$ SPL on the façade are represented for the standard façade $(\Delta x \times \Delta y=4 \mathrm{~m}$ $\times 3 \mathrm{~m}$ ) using the same calculation procedure with EASE as in Fig. 11, but here considering both direct levels and the effect of reflection both in the façade and in the ground. It is assumed that 

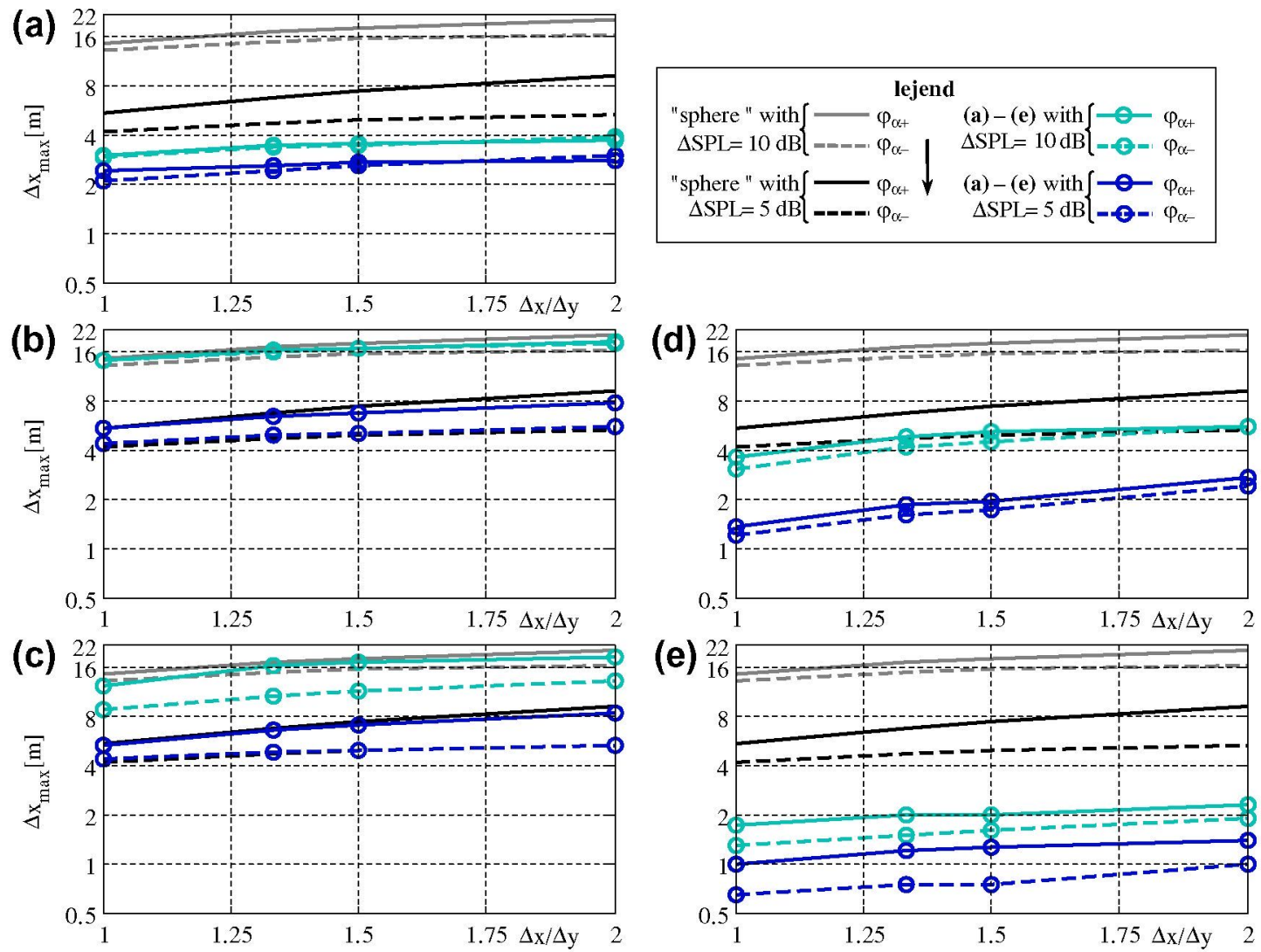

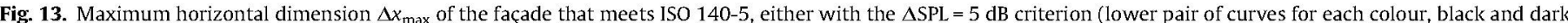

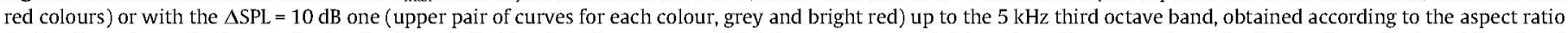

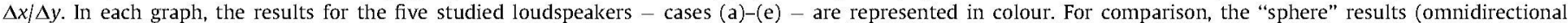

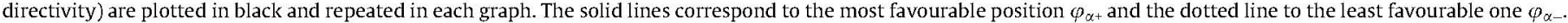

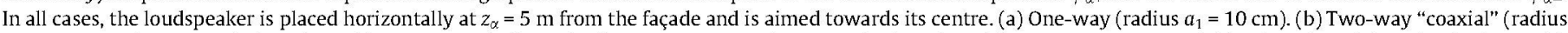

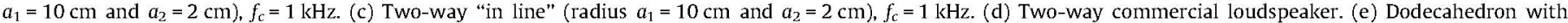
loudspeakers of radius $6 \mathrm{~cm}$.

Table 1

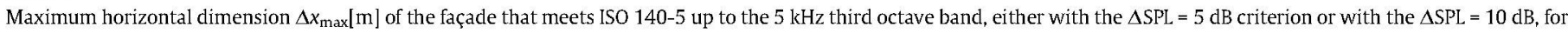

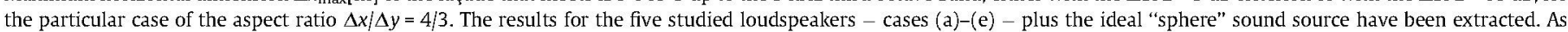
previously, $\Delta x_{\max }$ is shown for the most and the least favourable positions for the case of $\Delta x / \Delta y=4 / 3$, that is $\varphi_{\alpha_{+}}=-90^{\circ}$ and $\varphi_{\alpha-}=-36.9^{\circ}$.

\begin{tabular}{|c|c|c|c|c|c|c|c|c|c|c|c|c|}
\hline \multirow[b]{2}{*}{$\triangle \mathrm{SPL}=$} & \multicolumn{2}{|c|}{ "Sphere" } & \multicolumn{2}{|c|}{ (a) One-way } & \multicolumn{2}{|c|}{ (b) Two-way "coaxial" } & \multicolumn{2}{|c|}{ (c) Two-way "in line" } & \multicolumn{2}{|c|}{ (d) Two-way commercial } & \multicolumn{2}{|c|}{ (e) Dodecahedron } \\
\hline & $5 \mathrm{~dB}$ & $10 \mathrm{~dB}$ & $5 \mathrm{~dB}$ & $10 \mathrm{~dB}$ & $5 \mathrm{~dB}$ & $10 \mathrm{~dB}$ & $5 \mathrm{~dB}$ & $10 \mathrm{~dB}$ & $5 \mathrm{~dB}$ & $10 \mathrm{~dB}$ & $5 \mathrm{~dB}$ & $10 \mathrm{~dB}$ \\
\hline$\varphi_{\alpha+}=-90^{\circ}$ & $6.80 \mathrm{~m}$ & $17.1 \mathrm{~m}$ & $2.60 \mathrm{~m}$ & $3.40 \mathrm{~m}$ & $6.45 \mathrm{~m}$ & $16.20 \mathrm{~m}$ & $6.50 \mathrm{~m}$ & $16.30 \mathrm{~m}$ & $1.85 \mathrm{~m}$ & $4.85 \mathrm{~m}$ & $1.2 \mathrm{~m}$ & $2.00 \mathrm{~m}$ \\
\hline$\varphi_{\alpha+}=-36.9^{\circ}$ & $4.75 \mathrm{~m}$ & $14.8 \mathrm{~m}$ & $2.40 \mathrm{~m}$ & $3.35 \mathrm{~m}$ & $4.90 \mathrm{~m}$ & $16.00 \mathrm{~m}$ & $4.80 \mathrm{~m}$ & $10.65 \mathrm{~m}$ & $1.60 \mathrm{~m}$ & $4.20 \mathrm{~m}$ & $0.75 \mathrm{~m}$ & $1.50 \mathrm{~m}$ \\
\hline
\end{tabular}

the loudspeaker is located at $25 \mathrm{~cm}$ from the ground and that the wall and ground have an absorption coefficient $\beta=80 \%$ for all frequencies. As expected, the situation is much worse than when only the direct sound field is considered, even if the absorption coefficient of walls and ground is relatively high, as has been applied in this simulation.

\section{Conclusions}

Several conclusions can be drawn from this work.

First, the ISO 140-5 requirements on the sound source are not so easily accomplished. The ideal omnidirectional source has a priori the best directivity because it produces relatively small direct acoustic level variation on the façade. As shown in Fig. 5, the omnidirectional source only fails to comply with the ISO standard conditions for very large façades, both with the $\triangle \mathrm{SPL}=5 \mathrm{~dB}$ and $\Delta \mathrm{SPL}=10 \mathrm{~dB}$ criteria. In any case, there are certain geometric conditions for which the measurements produce higher acoustic uniformity on the façade; that is, the loudspeaker placed at the most favourable position $\varphi_{\alpha^{+}}=-90^{\circ}$, and radiating over long façades, with high aspect ratios $\Delta x / \Delta y$, are the preferred geometric conditions.

Second, the source directivity always seems to be a detrimental factor. The source directivity may be thought to be able to correct the excess in the points near the emitter, but nevertheless, no advantages have been found over the level distribution on the façade from directive sources with respect to omnidirectional ones. 


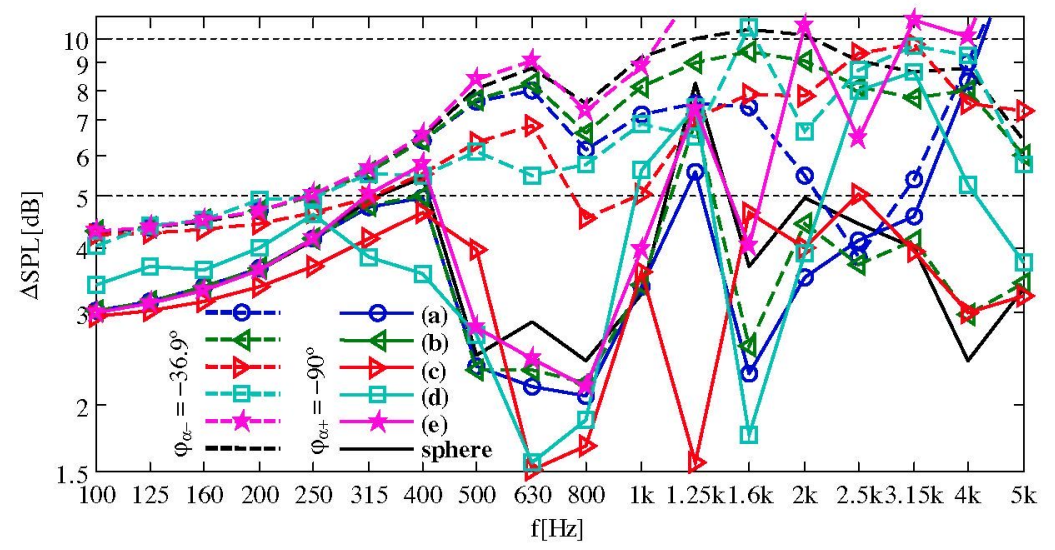

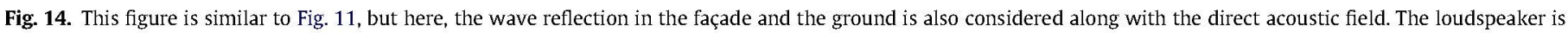
located $25 \mathrm{~cm}$ from the ground, and the absorption coefficient is $\beta=80 \%$ both for the wall and the ground at all frequencies.

Some tests have been implemented (data not shown) that slightly refocus the sound source to improve differences without positive results. In conclusion, omnidirectional or nearly omnidirectional sources are preferred over directive ones.

According to the above conclusions, the ideal source would be very small, which in theory can be almost omnidirectional up to $5 \mathrm{kHz}$, the limit frequency for the ISO standard. However, as is widely known, a small source is unable to radiate low frequency with acceptable levels, which leads to the choice of two-way loudspeakers. Finally, using theoretical models, the configuration "coaxial" has been found to be the best because it slightly overcomes the two-way loudspeaker with an "in line" arrangement. Horn-type high-frequency radiators are not usually the best choice. Dodecahedron sources, at least with the standard size and configuration as in the actual models used today, are strongly discouraged because their directivity becomes highly lobulated from $1 \mathrm{kHz}$.

On the other hand, wave reflections on the ground and walls were found to have a highly harmful effect on the façade acoustic levels, even when considering relatively high absorption coefficients. This result means that it is inadvisable to verify the source directivity in poorly controlled acoustic conditions (e.g., outdoors) where the ground absorption coefficient is unknown. Instead, it is recommended that the source directivity be measured in an anechoic room and that the acoustic levels be calculated on the façade according to the theoretical study proposed in this paper, or with a computational aid such as EASE. A procedure for verification of sound sources based on these simulations and in accordance with paragraph 4.2 of the ISO $140-5$ standard has been designed and successfully tested by the authors [9].

One of the objectives of this study was to establish a relationship between the source directivity and the level distribution on the façade. This relationship is shown in Fig. 8 with the pistons and the $-6 \mathrm{~dB}$ coverage angle, $\theta_{L(-6 \mathrm{~dB}}^{\prime}$. Although these results can provide certain guidelines for sources with piston-type directivity, the relationships shown in Fig. 8 should not be generalised to real sources with complicated directivity patterns and with no cylindrical symmetry. That is, for verifying whether a sound source fulfils the ISO 140-5 restrictions is insufficient to analyse its coverage angle, and it would be necessary to calculate or simulate its directivity function and to project it onto the façade in combination with the distance effects, including air absorption [9], as has been discussed in this paper.

Finally, as a future line of work, the combination of the calculated sound levels outside the façade and in the measurement microphone, according to ISO 140-5, with the mechanical and acoustic properties of the façade and the receiving room in order to simulate the transmitted levels and to study the influence of each element in the insulation of the façade, obtained in the ISO standard way [10], may be very interesting.

\section{Appendix A. Nearest and farthest point from the sound source within the façade}

Assuming that the source can be placed only at the fourth quadrant of the coordinate system, as shown in Fig. 1 (i.e., $\left.-90^{\circ}<\varphi_{\alpha}<0^{\circ}\right)$, the closest and farthest points will be determined respectively by the smallest and largest circumferences projected from the loudspeaker onto the façade that contact this façade in one single point (tangent).

Fig. A1 shows the position of point 0 , the closest from the source within the façade, and the circumferences of the smallest radius that can be drawn from the loudspeaker horizontal projection over the vertical plane contacting with point 0 . If that horizontal projection falls away from the façade (more typical for small samples), then point 0 can be easily deduced as being located at the following positions:

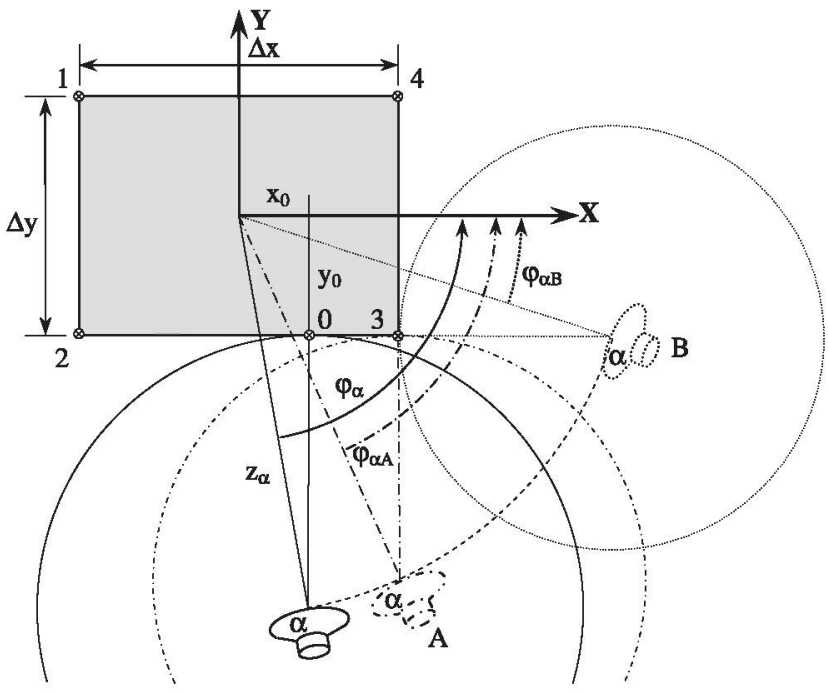

Fig. A1. Location of point 0 , the closest from the source within the façade. The smallest radius circumferences that can be drawn from the projection of the loudspeaker on the façade that make contact with the sample at point 0 are plotted for three loudspeaker positions. The angle $\varphi_{\alpha \mathrm{A}}$ corresponds to the loudspeaker placed just below corner 3 . The angle $\varphi_{\alpha \mathrm{B}}$ corresponds to the source on the horizontal of corner 3 . 
- at the bottom side of the façade for the loudspeaker placed in the interval $-90^{\circ}<\varphi_{\alpha}<\varphi_{\alpha A}$, with $\varphi_{\alpha A}$ as the angle corresponding to the source position when it is placed just below point 3 ,

- just at point 3 for the loudspeaker in the interval $\varphi_{\alpha A}<\varphi_{\alpha}<$ $\varphi_{\alpha B}$, with $\varphi_{\alpha B}$ as the angle for the emitter position when placed at the point 3 level and finally,

- at the right side of the façade when the loudspeaker is in the interval $\varphi_{\alpha A}<\varphi_{\alpha}<0^{\circ}$,

- conversely, if the loudspeaker horizontal projection falls on the façade, then the nearest point 0 is precisely that projection of the loudspeaker on the façade.

Analytically, the coordinates of point 0 , which is the closest to the loudspeaker, will be:

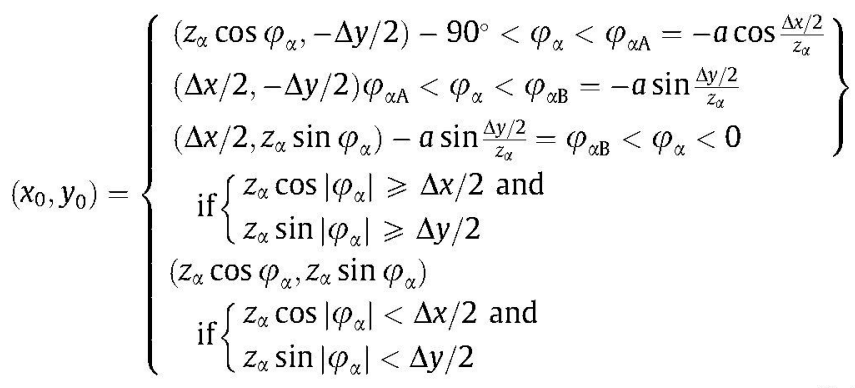

In (A.1), the first three sets of coordinates correspond to the case where the loudspeaker is projected outside the façade, most often with small surfaces.

The expression (A.1) particularised to the standard façade of $4 \mathrm{~m} \times 3 \mathrm{~m}$ and the sound source at a distance from this façade of $z_{\alpha}=5 \mathrm{~m}$ will be:

$$
\left(x_{0}, y_{0}\right)= \begin{cases}\left(5 \cos \varphi_{\alpha},-3 / 2\right) & -90^{\circ}<\varphi_{\alpha}<\varphi_{\alpha \mathrm{A}}=-66.4^{\circ} \\ (2,-3 / 2) & \varphi_{\alpha \mathrm{A}}<\varphi_{\alpha}<\varphi_{\alpha \mathrm{B}}=-17.5^{\circ} \\ \left(2,5 \sin \varphi_{\alpha}\right) & -17.5^{\circ}=\varphi_{\alpha \mathrm{B}}<\varphi_{\alpha}<0^{\circ} .\end{cases}
$$

The farthest point from the loudspeaker will be that crossed by the circumference of the greatest radius drawn from the loudspeaker projection on the vertical plane contacting some façade point. Clearly, this point is point 1 (top left corner), regardless of the loud-

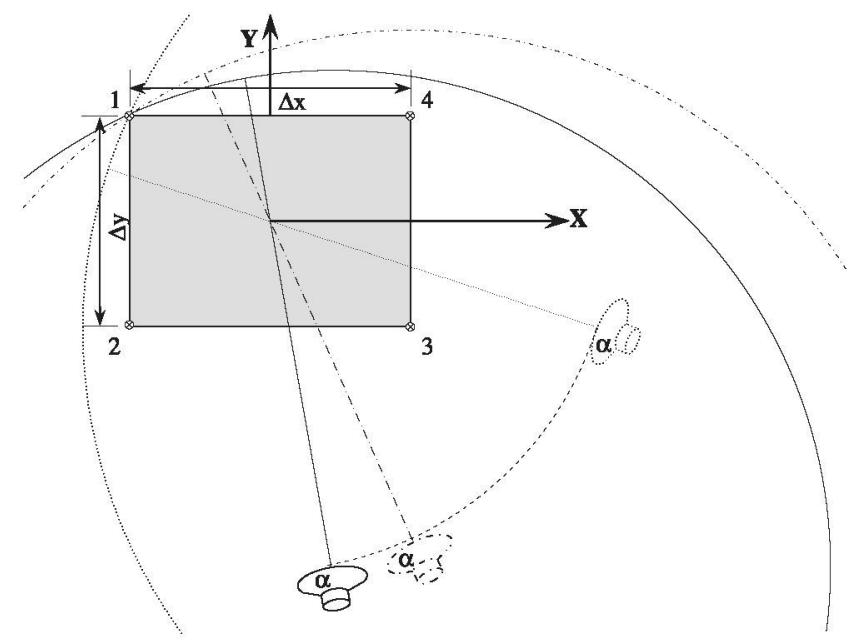

Fig. A2. Location of the farthest point from the source within the façade. For the three positions of the loudspeaker as in Fig. A1, the circumference of the largest radius that can be drawn from the horizontal projection of the loudspeaker contacting the façade is shown. This point is always the top left corner or point 1 for any value $\varphi_{\alpha}$, provided that the sound source is placed in the fourth quadrant $\left(-90^{\circ}<\varphi_{\alpha}<0^{\circ}\right)$. speaker angle $\varphi_{\alpha}$. Fig. A2 shows the location of the farthest point from the source within the façade. For the three positions of the source in Fig. A1, the circumference of the largest radius that can be drawn from the loudspeaker horizontal projection that contacts some point of the sample is also shown. Thus, the top left corner (point 1) will invariably be the farthest one from the loudspeaker, provided that it is placed in the fourth quadrant $\left(-90^{\circ}<\varphi_{\alpha}<0^{\circ}\right)$.

That is, the coordinates of point 1 , the farthest from the loudspeaker, will be:

$\left(x_{1}, y_{1}\right)=(-\Delta x / 2, \Delta y / 2) \quad-90^{\circ}<\varphi_{\alpha}<0^{\circ}$.

Thus, the expression (A.3) particularised to the standard façade of $4 \mathrm{~m} \times 3 \mathrm{~m}$ and a sound source at $z_{\alpha}=5 \mathrm{~m}$ will be:

$\left(x_{1}, y_{1}\right)=(-2,3 / 2) \quad-90^{\circ}<\varphi_{\alpha}<0^{\circ}$.

\section{Appendix B. Distance " $r$ " from the loudspeaker to the generic point $P(x, y)$ within the façade vs. the source position $\left(r_{\alpha}, \varphi_{\alpha}\right)$}

As depicted in Fig. 1, and because the elevation angle is fixed at $\theta_{\alpha}=45^{\circ}$, the distance " $r$ " to the generic point $P(x, y)$ on the façade from the source located at a the generic point $\left(r_{\alpha}, \varphi_{\alpha}, 45^{\circ}\right)$ in spherical coordinates is given by the following expression:

$$
\begin{aligned}
r^{2}= & \left(r_{\alpha} \cdot \sin 45^{\circ} \cos \varphi_{\alpha}-x\right)^{2}+\left(r_{\alpha} \sin 45^{\circ} \sin \varphi_{\alpha}-y\right)^{2}+\left(r_{\alpha}\right. \\
& \left.\times \cos 45^{\circ}\right)^{2} \\
= & \left(z_{\alpha} \cos \varphi_{\alpha}-x\right)^{2}+\left(z_{\alpha} \sin \varphi_{\alpha}-y\right)^{2}+z_{\alpha}^{2} \\
= & x^{2}+y^{2}+2 z_{\alpha}^{2}-2 z_{\alpha}\left(x \cos \varphi_{\alpha}+y \sin \varphi_{\alpha}\right) .
\end{aligned}
$$

The expression (B.1) particularised to our standard façade of $4 \mathrm{~m} \times 3 \mathrm{~m}$ and with the loudspeaker at a distance from the façade of $z_{\alpha}=5 \mathrm{~m}$ will be:

$r^{2}=x^{2}+y^{2}+50-10\left(x \cdot \cos \varphi_{\alpha}+y \cdot \sin \varphi_{\alpha}\right)$.

\section{Appendix C. Angles of the generic façade points $P(x, y)$ relative to the loudspeaker coordinate system}

As the starting point, the loudspeaker is assumed to be horizontally placed, so the coordinate axes $\mathbf{X}^{\prime}$ (loudspeaker) and $\mathbf{X}$ (façade) are considered as coplanar. The loudspeaker axis lies in $\mathbf{Z}$ ' and points to the coordinate origin of the façade, "o". With these conditions, the elevation and azimuth angles of each façade pair $(x, y), \theta^{\prime}(x, y)$ and $\varphi^{\prime}(x, y)$, can be calculated.

C.1. Calculation of the elevation angle $\theta^{\prime}(x, y)$ for each façade point in the loudspeaker coordinate system

The elevation angle $\theta^{\prime}$ is that angle formed by the $\mathbf{Z}^{\prime}$ axis and the vector $\mathbf{r}$ as well as the angle formed by $\mathbf{r}_{\alpha}$ and $\mathbf{r}$ (Fig. 1 ). The angle formed by two lines defined by its direction cosines $l, m$ and $n$ is given by:

$\cos \theta^{\prime}=l_{z^{\prime}} l_{r}+m_{z^{\prime}} m_{r}+n_{z^{\prime}} n_{r}$,

where $l_{z^{\prime}}, m_{z^{\prime}}$ and $n_{z^{\prime}}$ are the direction cosines of the $\mathbf{Z}^{\prime}$ axis in the façade coordinate system:

$$
\begin{aligned}
& l_{z^{\prime}}=\frac{0-x_{\alpha}}{r_{\alpha}}=\frac{0-r_{\alpha} \sin \theta_{\alpha} \cos \varphi_{\alpha}}{r_{\alpha}}=-\sin \theta_{\alpha} \cos \varphi_{\alpha}=-\sin 45^{\circ} \cos \varphi_{\alpha}=-\frac{\cos \varphi_{\alpha}}{\sqrt{2}} \\
& m_{z^{\prime}}=\frac{0-y_{\alpha}}{r_{\alpha}}=\frac{0-r_{\alpha} \sin \theta_{\alpha} \sin \varphi_{\alpha}}{r_{\alpha}}=-\sin \theta_{\alpha} \sin \varphi_{\alpha}=-\sin 45^{\circ} \sin \varphi_{\alpha}=-\frac{\sin \varphi_{\alpha}}{\sqrt{2}} \\
& n_{z^{\prime}}=\frac{0-z_{\alpha}}{r_{\alpha}}=\frac{0-r_{\alpha} \cos \theta_{\alpha}}{r_{\alpha}}=-\cos \theta_{\alpha}=-\cos 45^{\circ}=-\frac{1}{\sqrt{2}}
\end{aligned}
$$

and $l_{r}, m_{r}$ and $n_{r}$ are the direction cosines of the vector $\mathbf{r}$ in the same reference system: 


$$
\begin{aligned}
& l_{r}=\frac{x-x_{\alpha}}{r}=\frac{x-r_{\alpha} \sin \theta_{\alpha} \cos \varphi_{\alpha}}{r}=\frac{x-r_{\alpha} \sin 45^{\circ} \cos \varphi_{\alpha}}{r}=\frac{x-r_{\alpha} \cos \varphi_{\alpha} / \sqrt{2}}{r}=\frac{x-z_{\alpha} \cos \varphi_{\alpha}}{r} \\
& m_{r}=\frac{y-y_{\alpha}}{r}=\frac{y-r_{\alpha} \sin \theta_{\alpha} \sin \varphi_{\alpha}}{r}=\frac{y-r_{\alpha} \sin 45^{\circ} \operatorname{sen} \varphi_{\alpha}}{r}=\frac{y-z_{\alpha} \sin \varphi_{\alpha}}{r} \\
& n_{r}=\frac{0-z_{\alpha}}{r}=-\frac{r_{\alpha} \cos \theta_{\alpha}}{r}=-\frac{r_{\alpha} \cos 45^{\circ}}{r}=-\frac{z_{\alpha}}{r} .
\end{aligned}
$$

Moreover, the distance " $r$ " from the loudspeaker to the generic façade point $P(x, y)$ previously obtained in (B.1), can be also considered as the coordinate " $r$ " of that point with respect to the coordinate system of the loudspeaker, as follows:

$$
\begin{aligned}
r^{\prime} & \equiv r=\sqrt{\left(x-x_{\alpha}\right)^{2}+\left(y-y_{\alpha}\right)^{2}+z_{\alpha}^{2}} \\
& =\sqrt{\left(x-r_{\alpha} \sin \theta_{\alpha} \cos \varphi_{\alpha}\right)^{2}+\left(y-r_{\alpha} \sin \theta_{\alpha} \sin \varphi_{\alpha}\right)^{2}+r_{\alpha}^{2} \cos ^{2} \theta_{\alpha}} \\
& =\sqrt{x^{2}+y^{2}+r_{\alpha}^{2}-r_{\alpha} \sqrt{2}\left(x \cos \varphi_{\alpha}+y \sin \varphi_{\alpha}\right)} \\
& =\sqrt{x^{2}+y^{2}+2 z_{\alpha}^{2}-z_{\alpha}\left(x \cos \varphi_{\alpha}+y \sin \varphi_{\alpha}\right)} .
\end{aligned}
$$

Thus, by replacing (C.2), (C.3), and (C.4) in (C.1):

$\cos \theta^{\prime}=\frac{2 z_{\alpha}-x \cos \varphi_{\alpha}-y \sin \varphi_{\alpha}}{\sqrt{2 x^{2}+2 y^{2}+4 z_{\alpha}^{2}-4 z_{\alpha}\left(x \cos \varphi_{\alpha}+y \sin \varphi_{\alpha}\right)}}$.

The expression (C.5) particularised for $z_{\alpha}=5 \mathrm{~m}$ is:

$\cos \theta^{\prime}=\frac{10-x \cos \varphi_{\alpha}-y \sin \varphi_{\alpha}}{\sqrt{2 x^{2}+2 y^{2}+10-20\left(x \cos \varphi_{\alpha}+y \sin \varphi_{\alpha}\right)}}$.

C.2. Calculation of the azimuth angle $\varphi^{\prime}(x, y)$ for each façade point in the loudspeaker coordinate system

The angle $\varphi^{\prime}$ corresponds to the azimuth spherical coordinate for the point $P(x, y)$ as in Fig. 1 and expressed in the loudspeaker reference system, $\mathbf{X}^{\prime}, \mathbf{Y}^{\prime}$ and $\mathbf{Z}^{\prime}$, namely:

$\tan \varphi^{\prime}=\frac{y^{\prime}}{x^{\prime}}$

where $\left(x^{\prime}, y^{\prime}\right)$ are the coordinates of point $P$ in the loudspeaker coordinate system. To obtain $\left(x^{\prime}, y^{\prime}\right)$ vs. $(x, y)$, the change of coordinates is applied using the following expression:

$x^{\prime}=l_{x^{\prime}}\left(x-x_{\alpha}\right)+m_{x^{\prime}}\left(y-y_{\alpha}\right)+n_{x^{\prime}}\left(z-z_{\alpha}\right)=l_{x^{\prime}}\left(x-x_{\alpha}\right)+m_{x^{\prime}}\left(y-y_{\alpha}\right)-n_{x^{\prime}} z_{\alpha}$ $y^{\prime}=l_{y^{\prime}}\left(x-x_{\alpha}\right)+m_{y^{\prime}}\left(y-y_{\alpha}\right)+n_{y^{\prime}}\left(z-z_{\alpha}\right)=l_{y^{\prime}}\left(x-x_{\alpha}\right)+m_{y^{\prime}}\left(y-y_{\alpha}\right)-n_{y^{\prime}} z_{\alpha}$,

with $l_{x^{\prime}}, m_{x^{\prime}}$ and $n_{x^{\prime}}$ being the direction cosines of the $\mathbf{X}^{\prime}$ axis in the façade reference system and $l_{y^{\prime}}, m_{y^{\prime}}$ and $n_{y^{\prime}}$ being the direction cosines of $\mathbf{Y}^{\prime}$ in the same coordinate system.

To calculate $I_{X^{\prime}}, m_{X^{\prime}}$ and $n_{X^{\prime}}$, it must be considered that the $\mathbf{X}^{\prime}$ axis is horizontal (the loudspeaker is placed horizontally and aiming to the façade centre) and, therefore, perpendicular to Y. Then:

$m_{x^{i}}=0$

In addition, the $\mathbf{X}^{\prime}$ axis is also perpendicular to $\mathbf{Z}^{\prime}$, so the cosine of the angle between $\mathbf{X}^{\prime}$ and $\mathbf{Z}^{\prime}$ is zero, and the sum of products between the respective direction cosines:

$$
\begin{aligned}
\cos 90^{\circ} & =l_{z^{\prime}} l_{x^{\prime}}+m_{z^{\prime}} m_{x^{\prime}}+n_{z^{\prime}} n_{x^{\prime}}=0 \Rightarrow n_{x^{\prime}}=-\frac{l_{z^{\prime}} l_{x^{\prime}}}{n_{z^{\prime}}} \\
& =-l_{x^{\prime}} \cos \varphi_{\alpha} .
\end{aligned}
$$

Furthermore, it should be verified that the quadratic sum of the direction cosines of the axes must be unitary, that is:

$$
\begin{aligned}
l_{x^{\prime}}^{2}+m_{x^{\prime}}^{2}+n_{x^{\prime}}^{2} & =1 \Rightarrow l_{x^{\prime}}^{2}+l_{x^{\prime}}^{2} \cos ^{2} \varphi_{\alpha}=1 \Rightarrow l_{x^{\prime}} \\
& = \pm \frac{1}{\sqrt{1+\cos ^{2} \varphi_{\alpha}}} .
\end{aligned}
$$

When replacing (C.11) in (C.10), it becomes:

$n_{x^{\prime}}=\mp \frac{\cos \varphi_{\alpha}}{\sqrt{1+\cos ^{2} \varphi_{\alpha}}}$.

To calculate the remaining direction cosines $l_{y^{\prime}}, m_{y^{\prime}}$ and $n_{y^{\prime}}$, it should be noted the $\mathbf{Y}^{\prime}$ axis is perpendicular to $\mathbf{X}^{\prime}$, that is:

$$
\begin{aligned}
\cos 90^{\circ} & =l_{x^{\prime}} l_{y^{\prime}}+n_{x^{\prime}} n_{y^{\prime}}=0 \\
& \Rightarrow \pm \frac{l_{y^{\prime}}}{\sqrt{1+\cos ^{2} \varphi_{\alpha}}} \mp \frac{n_{y^{\prime}} \cos \varphi_{\alpha}}{\sqrt{1+\cos ^{2} \varphi_{\alpha}}}=0 \Rightarrow l_{y^{\prime}} \\
& =n_{y^{\prime}} \cos \varphi_{\alpha} .
\end{aligned}
$$

Moreover, $\mathbf{Y}^{\prime}$ is perpendicular to $\mathbf{Z}^{\prime}$, therefore:

$$
\begin{aligned}
\cos 90^{\circ} & =l_{z^{\prime}} l_{y^{\prime}}+m_{z^{\prime}} m_{y^{\prime}}+n_{z^{\prime}} n_{y^{\prime}}=0 \\
& \Rightarrow-\frac{\cos \varphi_{\alpha}}{\sqrt{2}} n_{y^{\prime}} \cos \varphi_{\alpha}-\frac{\sin \varphi_{\alpha}}{\sqrt{2}} m_{y^{\prime}}-\frac{1}{\sqrt{2}} n_{y^{\prime}}=0 \\
& \Rightarrow m_{y^{\prime}}=-\frac{1+\cos ^{2} \varphi_{\alpha}}{\sin \varphi_{\alpha}} n_{y^{\prime}} .
\end{aligned}
$$

Also, it must be verified:

$$
\begin{aligned}
l_{y^{\prime}}^{2}+m_{y^{\prime}}^{2}+n_{y^{\prime}}^{2} & =1 \Rightarrow n_{y^{\prime}}^{2} \cos ^{2} \varphi_{\alpha}+\frac{\left(1+\cos ^{2} \varphi_{\alpha}\right)^{2}}{\operatorname{sen}^{2} \varphi_{\alpha}} n_{y^{\prime}}^{2}+n_{y^{\prime}}^{2} \\
& =1 \Rightarrow n_{y^{\prime}}= \pm \frac{1}{\sqrt{\cos ^{2} \varphi_{\alpha}+\frac{\left(1+\cos ^{2} \varphi_{\alpha}\right)^{2}}{\operatorname{sen}^{2} \varphi_{\alpha}}+1}} \\
& = \pm \frac{\operatorname{sen} \varphi_{\alpha}}{\sqrt{4-2 \operatorname{sen}^{2} \varphi_{\alpha}}}= \pm \frac{\operatorname{sen} \varphi_{\alpha}}{\sqrt{2+2 \cos ^{2} \varphi_{\alpha}}}
\end{aligned}
$$

Now, substituting back (C.15) in (C.13) and (C.14) yields:

$l_{y^{\prime}}= \pm \frac{\sin \varphi_{\alpha} \cos \varphi_{\alpha}}{\sqrt{2+2 \cos ^{2} \varphi_{\alpha}}}$

$m_{y^{\prime}}=\mp \sqrt{\frac{\left(1+\cos ^{2} \varphi_{\alpha}\right)}{2}}$

Finally, substituting (C.9), (C.11), (C.12), (C.15), (C.16), and (C.17) in (C.8):

$$
\begin{aligned}
\tan \varphi^{\prime}= & \frac{y^{\prime}}{x^{\prime}}=\frac{l_{y^{\prime}}\left(x-x_{\alpha}\right)+m_{y^{\prime}}\left(y-y_{\alpha}\right)-n_{y^{\prime}} z_{\alpha}}{l_{x^{\prime}}\left(x-x_{\alpha}\right)+m_{x^{\prime}}\left(y-y_{\alpha}\right)-n_{x^{\prime}} z_{\alpha}} \\
= & \frac{ \pm \frac{\sin \varphi_{\alpha} \cos \varphi_{\alpha}}{\sqrt{2+2 \cos ^{2} \varphi_{\alpha}}}\left(x-z_{\alpha} \cos \varphi_{\alpha}\right) \mp \sqrt{\frac{\left(1+\cos ^{2} \varphi_{\alpha}\right)}{2}}\left(y-z_{\alpha} \sin \varphi_{\alpha}\right) \mp \frac{z_{\alpha} \sin \varphi_{\alpha}}{\sqrt{2+2 \cos ^{2} \varphi_{\alpha}}}}{ \pm \frac{1}{\sqrt{1+\cos ^{2} \varphi_{\alpha}}}\left(x-z_{\alpha} \cos \varphi_{\alpha}\right) \pm \frac{z_{\alpha} \cos \varphi_{\alpha}}{\sqrt{1+\cos ^{2} \varphi}}} \\
= & \frac{ \pm x \sin \varphi_{\alpha} \cos \varphi_{\alpha} \mp y\left(1+\cos ^{2} \varphi_{\alpha}\right) \pm z_{\alpha} \cos ^{2} \varphi_{\alpha} \sin \varphi_{\alpha} \mp z_{\alpha} \sin \varphi_{\alpha}}{ \pm x \sqrt{2}} \\
& =\frac{\sin \varphi_{\alpha} \cos \varphi_{\alpha}}{\sqrt{2}}-\frac{y\left(1+\cos ^{2} \varphi_{\alpha}\right)}{x \sqrt{2}}
\end{aligned}
$$

where $x_{\alpha}=z_{\alpha} \cos \varphi_{\alpha}$ and $y \alpha=z_{\alpha} \sin \varphi_{\alpha}$ have been considered, according to Fig. 1. The angle $\varphi^{\prime}$ can be considered independent of the loudspeaker distance to the façade, $z_{\alpha}$, provided that the geometric conditions of ISO $140-5$ are met.

\section{References}

[1] ISO 140-5:1998. Acoustics - measurement of sound insulation in buildings and of building elements. Part 5: field measurements of airborne sound insulation of façade elements and façades; 1998. 
[2] Rasmussen B, Rindel JH. Sound insulation between dwellings - descriptor applied in building regulations in Europe. Appl Acoust 2010;71(3):171-80.

[3] Rasmussen B. Sound insulation between dwellings - requirements in building regulations in Europe. Appl Acoust 2010;71(4):373-85

[4] Beranek LL. Acoustics. McGraw Hill; 1954

[5] EASE, Enhanced acoustic simulator for engineers. Information available in <http://www.ease.afmg.eu/>.

[6] Feistel S, Ahnert W. Modeling of loudspeaker systems using high-resolution data. J Audio Eng Soc 2007;55:571-97.

[7] Feistel S, Ahnert W, Hughes C, Olson B. Simulating the directivity behavior of loudspeakers with crossover filters. Paper 7254, 123rd convention, audio engineering society, New York, USA; October 5-8, 2007.
[8] Hopkins C, Lam Y. Sound fields near building facades - comparison of finite and semi-infinite reflectors on a rigid ground plane. Appl Acoust $2009 ; 70(2): 300-8$.

[9] Sánchez Bote JL, Gómez Alfageme JJ. Procedimiento para la medición y verificación de la directividad y la cobertura de una fuente sobre un elemento de fachada de acuerdo a la norma ISO 140-5, 42 Congreso Español de Acústica, Tecniacústica 2011, Cáceres, Spain; October 26-28, 2011. 11 pp [in Spanish].

[10] Pedrero González A, Sănchez Bote JL, Ulin Nabatov V, Diaz Sanchidrián C. Influence of loudspeaker directivity on the measurement uncertainty of the acoustic testing of façades. 40th International congress and exposition on noise control engineering (Inter Noise 2011), Osaka, Japan; September 4-7, 2011. 6 pp. 